\title{
A Review of the Effect of Processing Variables on the Fabrication of Electrospun Nanofibers for Drug Delivery Applications
}

\author{
Viness Pillay, ${ }^{1}$ Clare Dott, ${ }^{1}$ Yahya E. Choonara, ${ }^{1}$ Charu Tyagi, ${ }^{1}$ Lomas Tomar, ${ }^{1}$ \\ Pradeep Kumar, ${ }^{1}$ Lisa C. du Toit, ${ }^{1}$ and Valence M. K. Ndesendo ${ }^{2}$ \\ ${ }^{1}$ Department of Pharmacy and Pharmacology, Faculty of Health Sciences, University of the Witwatersrand, \\ 7 York Road, Parktown, Johannesburg 2193, South Africa \\ ${ }^{2}$ School of Pharmacy and Pharmaceutical Sciences, St. John's University of Tanzania, Dodoma, Tanzania \\ Correspondence should be addressed to Viness Pillay; viness.pillay@wits.ac.za
}

Received 21 November 2012; Accepted 20 December 2012

Academic Editor: Lianjun Wang

Copyright (C) 2013 Viness Pillay et al. This is an open access article distributed under the Creative Commons Attribution License, which permits unrestricted use, distribution, and reproduction in any medium, provided the original work is properly cited.

\begin{abstract}
Electrospinning is a fast emerging technique for producing ultrafine fibers by utilizing electrostatic repulsive forces. The technique has gathered much attention due to the emergence of nanotechnology that sparked worldwide research interest in nanomaterials for their preparation and application in biomedicine and drug delivery. Electrospinning is a simple, adaptable, cost-effective, and versatile technique for producing nanofibers. For effective and efficient use of the technique, several processing parameters need to be optimized for fabricating polymeric nanofibers. The nanofiber morphology, size, porosity, surface area, and topography can be refined by varying these parameters. Such flexibility and diversity in nanofiber fabrication by electrospinning has broadened the horizons for widespread application of nanofibers in the areas of drug and gene delivery, wound dressing, and tissue engineering. Drug-loaded electrospun nanofibers have been used in implants, transdermal systems, wound dressings, and as devices for aiding the prevention of postsurgical abdominal adhesions and infection. They show great promise for use in drug delivery provided that one can confidently control the processing variables during fabrication. This paper provides a concise incursion into the application of electrospun nanofibers in drug delivery and cites pertinent processing parameters that may influence the performance of the nanofibers when applied to drug delivery.
\end{abstract}

\section{Introduction}

Electrospinning is a simple and versatile technique that utilizes electrostatic forces to produce very fine fibers of polymer ranging from submicron to nanometer sizes. The technique can be applied to generate fibers of a wide array of polymer types-synthetic [1], natural [2], biodegradable $[3,4]$, nondegradable [5], or their blends $[3,6]$. Although there are other conventional techniques for polymeric fiber fabrication such as phase separation [7], self-assembly [8-10], template synthesis [11,12], and mechanical drawing [13], electrospinning has gained much importance and is the preferred technique since it is relatively ease, cost effective, requires simple tooling, and is applicable for producing ultrafine fibers with simple step-up production that is not easily achievable with other conventional fiber-forming techniques. During electrospinning, a high voltage electric field, is applied to the polymer liquid (a solution or melt) resulting in ejection of a continuous jet strand from the eluting nozzle that accelerates toward the oppositely charged grounded collector. In the absence of any electric field the polymer droplet is held at the capillary tip by surface tension of the liquid [14, 15]. Upon application of an electric field, as the surface tension is balanced by the electrostatic forces, the droplet elongates and develops into a cone known as a "Taylor Cone." When the strength of the electrical field is sufficient to overcome the surface tension of the liquid, a fine fiber jet is ejected from the tip of the Taylor Cone $[16,17]$. As the fiber jet travels through the atmosphere, the solvent evaporates and solid polymer fibers are deposited on a grounded collector as a mesh or scaffold. Figure 1 provides a schematic representation of a typical electrospinning process [18]. 


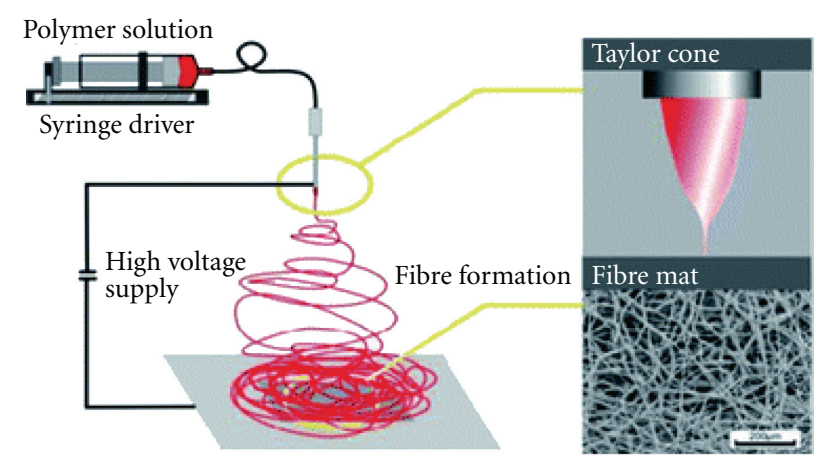

FIGURE 1: Schematic of a typical electrospinning setup showing the Taylor cone and SEM image of an electrospun mat. Adapted from Wallace et al. [18].

Fibers produced by conventional spinning methods are lengthened and thinned by being subjected to rheological, gravitational, tensile, inertial or aerodynamic forces [19]. In electrospinning, the induced charges under the applied field creates tensile forces in the axial direction of the flow of the polymer jet that results in fiber spinning $[19,20]$. Recent experiments and theoretical studies have revealed that the jet, after travelling a short distance, becomes unstable and starts whipping as a consequence of interaction between the applied external electrostatic field of attraction and the repulsive forces of the surface charges within the jet $[19,21]$. These interactions result in an increase in the fiber length and subsequent decrease in fiber diameter by many-fold compared to fibers produced by other methods. The added advantage's of electrospun fibers that render them with a wide range of applicability include the high surface to volume ratio, high porosity, and interconnected porous networks that are formed $[15,19,22]$.

The technique of electrospinning was first observed by Rayleigh in 1897 and investigated in detail by Zeleny in the early 1900s. However, electrospinning only surfaced as a feasible, small fiber diameter fabrication technique in 1934 after patented by Formhals [23-30]. Bherdwaj and Kundu [23] patented the electrospinning process and a device that consisted of a moveable fiber-collector, such as a mandrel, to collect fibers in a stretched form. However, the distance between the jet and collector was relatively small and the fibers were unable to dry completely before reaching the collector. This resulted in the fibers sticking to each other and to the collector surface [27]. Formhals [28], in his second patent, improved the drawbacks associated with his earlier apparatus. Follow-up patents of Formhals [29] pertained to improvements related to the control over the length of the produced fibers, formation of composite fibers by an electrospinning process that involved the deposition of spun fibers onto a base fiber [29], and increase of the fiber strength by drawing fibers through a rotating funnel [30].

After much fundamental research on the basics of the electrospinning process, researchers investigated the effects of various processing variables on the structural morphology and properties of electrospun fibers [14, 31, 32]. A deeper understanding of apparently relative correlations of various processing parameters and its impact on the fiber morphology and properties resulted in tailoring polymeric fibers of small diameter with desirable surface and bulk properties. This led to the evolvement of the application of electrospun nanofibers in diverse fields. In electronics, electrospun nanofibers have been used as anode material in lithium ion batteries [33, 34], as fine conducting fibers and magnetic materials [35-37], in electronic micro- and nanodevices [38, 39], and as optical and electrical nanomaterials [40]. Electrospun nanofibers have an application as a catalytic substrate due to their high surface area per unit mass [4143]. Electrospun nonwoven mats have long been used for air filtration and have been successfully fabricated as high performance air filters such as High Efficiency Particulate Air (HEPA) filters $[44,45]$ and filters with a high filtration efficiency and low air resistance [46].

Nanofibrous materials have also been investigated for their superior absorption and adsorption potential and may be applied for water absorption [47] and adsorption of pollutants such as toluene and benzene [48]. In addition, electrospun nanofibrous membranes have been employed in the fabrication of sensors for the detection of gases [49, 50], chemical substances such as urea [51], and drugs such as anticancer agents (e.g., daunorubicin) [52]. Biomedically, electrospun nanofibers have received much attention in the areas of drug delivery $[53,54]$, gene delivery $[55,56]$, prevention of postoperative adhesions [57], wound dressings $[58,59]$, and tissue engineering $[60,61]$. Tissue engineering applications of electrospun nanofibers include artificial vascular graft constructs $[1,62,63]$, as well as the engineering of neural tissue [64-66], tendon and ligament tissue [67, 68], and bone tissue $[69,70]$. This paper delves into the effects of processing parameters on electrospun nanofiber morphology and characteristics with emphasis on modulating the porosity and morphology of the fibers and mesh for pharmaceutical applications. In addition, a brief is provided on other related or potential applications of electrospun nanofibers.

\section{The Process of Electrospinning}

The process of electrospinning actually has its origin from the established process of electrospraying that utilizes the same principle of applying an electric potential to a polymer liquid and causing a jet of liquid to accelerate from a capillary tip toward an oppositely charged collector [23]. When the liquid to which the potential is applied has a low viscosity, the jet during its trajectory will break up due to the surface tension resulting in the formation of polymer droplets instead of fibers $[22,71]$. This is known as electrospraying and has application in the production of fine aerosol particles in the submicron size range and has practically been applied in pesticide and electrostatic sprayers $[72,73]$. However, if the liquid to which the potential is applied has a greater viscosity ( 1-200 poise), the jet will be less likely to break up because the greater viscoelastic forces would dampen the Rayleigh breaking mechanism as the so-called viscoelastic forces never act against the surface tension. It will then travel towards the grounded target in the form of a continuous jet finally depositing as nanofibers, and this constitutes electrospinning 
[23]. Apart from the surface tension and forces of the polymer solution, that is, electrospun, there are various other processing variables that are deciding factors for the final outcome of the process and the performance of the fabricated nanofibers.

Although the electrospinning process appears to be technically simple with ease of adaptability, a number of processing variables need to be regulated in order to generate nanofibers instead of droplets or beaded morphologies. The major challenge of the electrospinning process lies in the optimization of these parameters to achieve desirable nanofiber morphology and properties. These processing variables can be broadly termed as follows: the applied voltage, solution flow-rate, polymer concentration, solution viscosity, nature of solvent, solution conductivity, and the distance between the capillary and collector.

2.1. Effect of Changes in the Applied Voltage. Taylor [16] observed that the difference between the applied voltage that would cause a polymer drop to become unstable and that which would cause it to become conical in shape is very small. Any further increase in voltage beyond a critical value leads to the ejection of a polymer jet from the apex of the cone. This critical value of applied voltage varies with the type of polymer solution and there is an optimum range of the applied voltage or the electric field strength for a given polymer-solvent system within which nanofiber formation is desirable. An electric field that may be weaker or stronger than this critical value will result in beaded morphologies or even inhibit polymer jet initiation. In general, with an increase in the applied voltage beyond a critical value, the nanofiber diameter decreases initially and then increases after a definite point [74]. The initial decrease in nanofiber diameter is attributed to a higher degree of jet stretching in correlation to increased charge repulsion within the jet and a strong external electric field as a consequence of an increase in the applied voltage. In a study by Baumgarten [14], the jet length of a polymer solution at the optimal feed rate increased when the applied voltage was increased. At a capillaryto-collector gap of $50 \mathrm{~mm}$, the nanofiber diameter initially decreased to a minimum and then increased with increasing voltage. At a gap of $75 \mathrm{~mm}$, the decrease in nanofiber diameter to a minimum was not as apparent and the diameter increased slightly in response to an increase in the applied voltage. The optimum capillary feed rate also increased with an increase in the applied voltage.

Deitzel and coworkers [22] examined the effect of the applied voltage on a poly(ethylene oxide) (PEO)/water system. They showed that the applied voltage had a significant effect on the shape of the droplet from which the fiber jet originated. If all other variables except the applied voltage remain constant, an increase in the current would therefore generally reflect an increase in the flow-rate between the capillary and collector. An increase in the applied voltage in the range of $5.5-9.0 \mathrm{kV}$ resulted in an increase in the spinning current for the $\mathrm{PEO} /$ water system prototyped. At an applied voltage of $5.5 \mathrm{kV}$ the jet initiated from the apex of the Taylor Cone and nanofibers were regular free of defects.
Between $5.5 \mathrm{kV}$ and $7.0 \mathrm{kV}$, the current increased slowly with an increase in the applied voltage. At these lower voltages the nanofiber jet still ejected from the Taylor Cone at the bottom of the solution droplet (at the capillary end) and there were few bead defects associated with the nanofibers that had a cylindrical morphology. Greater than $7.0 \mathrm{kV}$ the current increased dramatically in response to the higher applied voltage and the jet was ejected from the Taylor Cone at the capillary tip. Beads on the nanofibers became more prevalent at an applied voltage of $7.0 \mathrm{kV}$ and their density increased further at $9.0 \mathrm{kV}$, when the jet was ejected from inside the capillary tip. Similar observations of an increase in bead defects with an increase in the applied voltage have been reported by other researchers $[75,76]$.

Meechaisue and coworkers [77] investigated the effect of the applied voltage on the electrospun nanofiber morphology of a poly(desaminotyrosyl-tyrosine ethyl ester carbonate) poly(DTE carbonate) solution at two different concentrations (15\% W/V and 20\% W/V). For the $15 \% \mathrm{~W} / \mathrm{V}$ polymer solution beaded nanofibers were mostly observed at applied voltages between $10.0 \mathrm{kV}$ and $15.0 \mathrm{kV}$, with the bead density decreasing as the voltage increased until smooth nanofibers were obtained at voltages between $20.0 \mathrm{kV}$ and $25.0 \mathrm{kV}$. At a polymer concentration of $20 \% \mathrm{~W} / \mathrm{V}$ the nanofibers were smooth at all applied voltages and the nanofiber diameter increased with increasing applied voltage in both cases for poly(DTE carbonate). The increase in nanofiber diameter was related to an increase in the flow-rate of the polymer solution as a result of the higher applied voltage.

In a similar study undertaken by Zong and coworkers [78], the effect of the applied voltage on the morphology of electrospun poly(D,L-lactic acid) (PDLLA) nanofibers was investigated. Jet formation was initiated at a voltage of $16.0 \mathrm{kV}$ and was not stable at voltages $<20.0 \mathrm{kV}$. At a voltage of $20.0 \mathrm{kV}$, the jet originated from the tip of the Taylor Cone and minimal bead formation was noted within the nanofiber structure. As the voltage was increased to $25.0 \mathrm{kV}$, the droplet volume of the polymer solution reduced and bead formation became more prevalent. Beads that were formed at $25.0 \mathrm{kV}$ had smaller diameters and higher density. When the applied voltage was further increased to $30.0 \mathrm{kV}$, the polymer droplet completely disappeared and the jet emerged from the tip of the capillary. The nanofibers obtained at this voltage had greater diameters and the formed beads became more spherical in shape.

2.2. Effect of Modifying the Solution Flow-Rate. The flowrate of the polymer solution through a capillary influences the nanofiber diameter, porosity, and geometry of the electrospun nanofibers. As observed by Deitzel and coworkers [22] as well as Zong and coworkers [78], in separate studies conducted to investigate the effect of an increase in the applied voltage, the volume of the polymer drop within the Taylor Cone decreased with an increase in voltage, eventually leading to the nanofiber jet being ejected from inside the capillary resulting in bead defects [74]. Therefore, in order to maintain the Taylor Cone shape at the capillary tip and avoid bead defects, a minimum flow-rate of the polymer is required in order to replace the solution that is lost when 
the nanofiber jet is ejected [26]. Megelski and coworkers [79] demonstrated an increase in diameter and pore size of electrospun polystyrene (from THF solution) nanofibers, to be associated with an increased flow-rate of the polymer solution. As the flow-rate increased, the available polymer volume was high which increased the nanofiber diameter along with an increase in pore size. When the flow-rate was too high, the nanofibers were unable to dry completely before reaching the collector and higher bead defects were therefore observed. Flattened ribbon-like nanofiber morphology may also result from incomplete drying of nanofibers due to a high flow-rate [79]. In another study by Zong and coworkers [78], it was shown that the lower the solution flow-rate, the smaller the diameter of the resultant electrospun nanofibers and bead defects. Beads that were formed on nanofibers that were electrospun using a higher solution flow-rate had larger diameters. This was attributed to the larger droplet at the end of the capillary, due to the higher flow-rate, resulting in the solution having a faster trajectory and resulting in incomplete drying and the formation of bead defects. Theron and coworkers [80] investigated the effect of solution flowrate on electric current and surface charge density. They found that an increase in flow-rate simultaneously increased the electric current and decreased the surfaces charge density.

\subsection{Effect of Polymer Concentration and Solution Viscosity.} The process of electrospinning is based on uniaxial stretching of a charged jet of polymer solution. At lower polymeric concentrations, due to the effect of the applied voltage and surface tension of the polymeric solution, the charged jet fragments into discrete droplets before reaching the collector $[81,82]$. At an increased polymeric concentration, as the viscosity increases the chain entanglement between polymeric chains improves and nanofibers are formed. The concentration of polymer in the solution to be electrospun thus has an effect on both the viscosity and surface tension of the liquid which ultimately decides the electrospinnability of the solution into nanofiber with increased diameters as the polymer concentration increases. However at concentrations beyond a certain limit, the viscosity of the solution becomes exceedingly high, disrupting the flow of the polymer solution through the capillary [81, 82].

Doshi and Reneker [15] investigated the effect of viscosity on the electrospinning of aqueous PEO solutions. They observed that nanofibers were formed from solutions with viscosities between 800 and $4000 \mathrm{cp}$. Below $800 \mathrm{cp}$, the nanofiber jet fragmented into droplets and $>4000 \mathrm{cp}$ nanofiber formation was difficult since the solution dehydrated at the tip of the capillary. Nanofiber formation therefore occurred in an optimum range of solution viscosity as dictated by an optimum polymer concentration. Furthermore, the effect of change in viscosity of PEO solutions on the appearance of bead defects in electrospun nanofibers was investigated by Fong and coworkers [83]. They reported that with an increase in solution viscosity, the bead diameter increased while the density of beads decreased. At greater viscosities the bead shape became less spherical and more spindle-like, resulting in nanofiber formation with diminished bead defects (Figure 2). A high surface tension of the solution at lower levels of polymer concentration caused the fiber jet to fragment into droplets. Viscoelastic forces competed with the surface tension in the nanofiber jet and an increase in viscosity therefore favored the formation of smooth nanofibers. In reports on electrospinning of polyacrylonitrile solutions, nanofiber formation occurred at a solution viscosity range of $1.7-215 \mathrm{cp}$. As the solution viscosity increased in this range, jet length and nanofiber diameter increased and the drop at the end of the capillary changed from hemispherical to conical shape [14]. However, the viscosities in the upper range resulted in incomplete drying of the polymer nanofiber thereby influencing the morphology of the formed nanofibers.

Meechaisue and coworkers [77] examined the effect of different concentrations of poly(DTE carbonate) on electrospun nanofiber formation. At a concentration of $5 \% \mathrm{~W} / \mathrm{V}(2.5 \mathrm{cp})$, mainly droplets were formed since the viscoelastic forces at this concentration were not sufficient to overcome the repulsive forces of charge, resulting in the fiber jet fragmenting into droplets. At higher polymer concentrations, the viscoelastic forces were sufficient to prevent fragmenting of the jet. Smooth nanofibers with bead defects were reported at a polymer concentration of $10 \% \mathrm{w} / \mathrm{v}(14.8 \mathrm{cp})$ and almost completely smooth nanofibers at $15 \% \mathrm{~W} / \mathrm{V}(50.5 \mathrm{cp})$. It was proposed that the repulsive forces from the charge within the fiber jet resulted in stretching and therefore a smaller nanofiber diameter at lower concentrations was observed. At a higher polymer concentration of $20 \% \mathrm{~W} / \mathrm{V}$ (196 cp), the viscoelastic force in the nanofiber jet resisted the stretching repulsive forces of charge, resulting in an increase in nanofiber diameter [77].

Deitzel and coworkers [22] also in their work on the electrospinning of aqueous $\mathrm{PEO}$ solutions reported the optimum polymer concentration range to be between 4 and $10 \% \mathrm{~W} / \mathrm{V}$ for nanofiber formation. Below this range surface tension was the detrimental factor and a combination of nanofibers and droplets were reported. At lower concentrations within the useful range, nanofibers had irregular morphologies along with bundles and junctions due to inadequate drying before reaching the collector. At higher concentrations within the useful range, the nanofibers were regular and cylindrical, with fewer junctions and bundles due to adequate drying before reaching the collector and the lower solvent content. Beyond the concentration of $10 \% \mathrm{w} / \mathrm{v}$, the flow of the polymer solution through the capillary could not be controlled due to cohesion, and nanofiber formation was inhibited at such a high viscosity [22]. Zong and coworkers [78] obtained similar patterns in attempting to electrospin PDLLA solutions of different concentrations and examining its effects on the resulting nanofiber morphology. They obtained a mixture of large bead defects and nanofibers on electrospinning PDLLA solutions of concentration $<20 \% \mathrm{~W} / \mathrm{V}$. At polymer concentrations $>40 \% \mathrm{~W} / \mathrm{V}$ the viscosity was too high to allow electrospinning. As the concentration and hence viscosity increased between these two extremes, the nanofibers produced were more uniform and the bead defects acquired larger diameters and became more spindle-shaped. It can therefore be concluded that viscosity and surface tension 


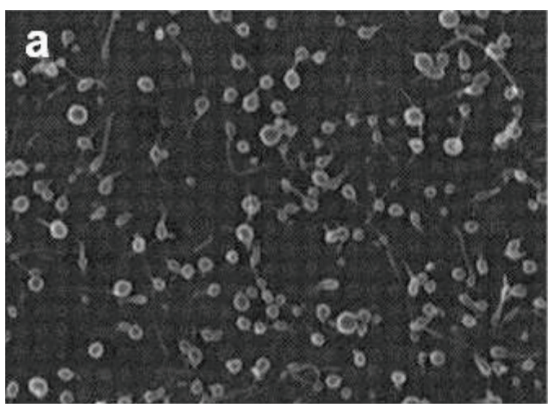

(a)

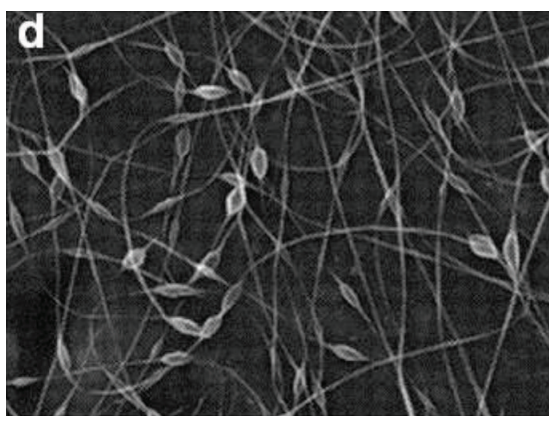

(d)

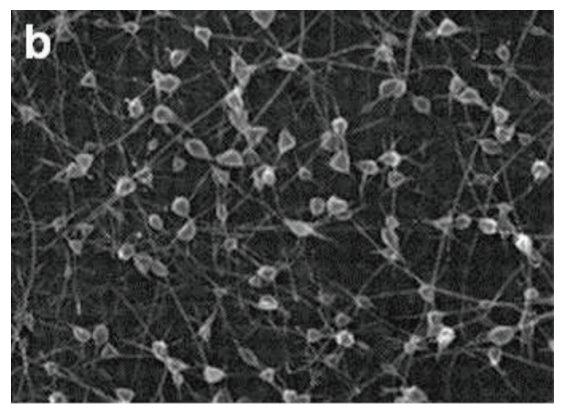

(b)

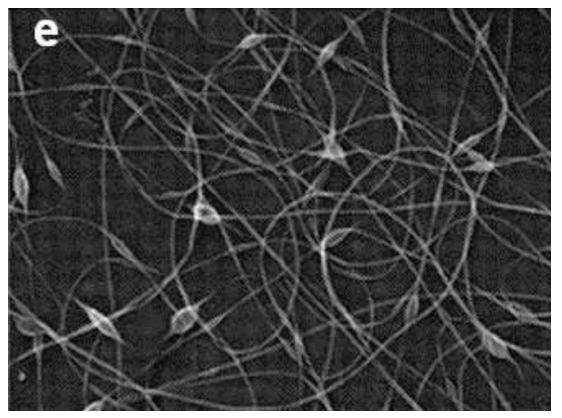

(e)

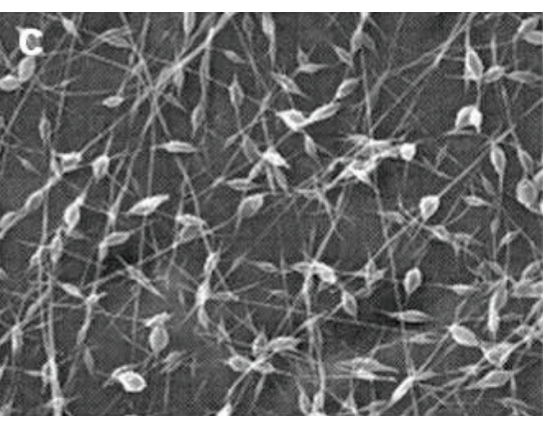

(c)

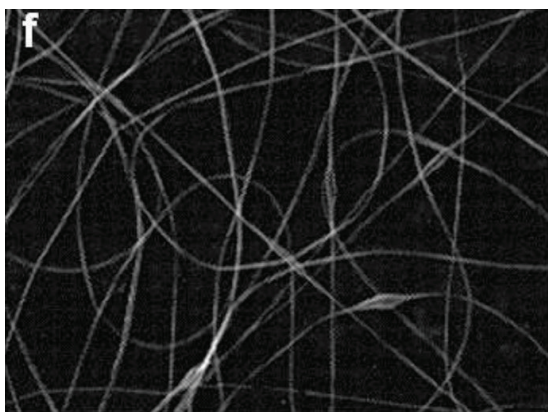

(f)

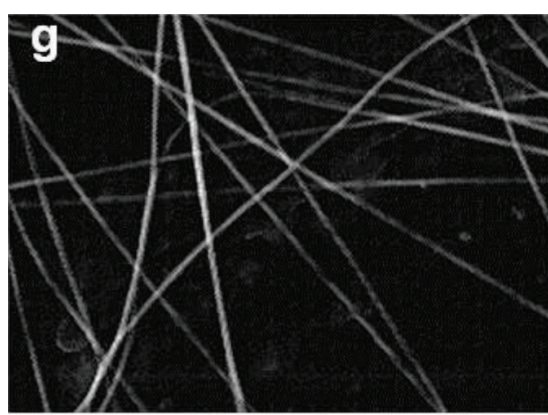

(g)

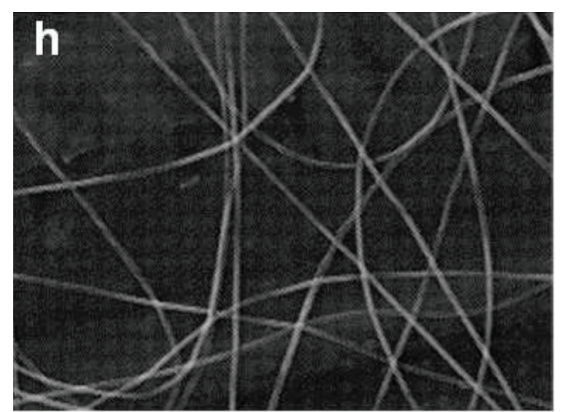

(h)

FIGURE 2: Morphology of electrospun PEO nanofibers (in water) at varying electrospinning solution viscosities. (a) 13 centipoise, (b) 32 centipoise, (c) 74 centipoise, (d) 160 centipoise, (e) 289 centipoise, (f) 527 centipoise, (g) 1250 centipoise, and (h) 1835 centipoise (electric field: $0.7 \mathrm{kV} / \mathrm{cm}$ ). Adapted from Fong et al. [83].

often determines the useful range of polymer concentrations in electrospinning for a given polymer.

2.4. Effect of Solvent Selection. The choice of solvent is critical for a particular polymer to solubilize and be transformed into nanofibers through electrospinning. Two important considerations for selecting a solvent are the solubility of the polymer in the solvent and the boiling point of the solvent as indicative of its volatility. Generally, volatile solvents are the preferred choice as they facilitate dehydration of the nanofibers during trajectory from the capillary tip to the collector surface owing to their lower boiling point and hence rapid evaporation rate. However, highly volatile solvents with very low boiling points should be avoided as they may evaporate at the capillary tip and thereby result in clogging and obstructing the flow-rate of the polymer solution. Solvents with high boiling points may not dehydrate completely prior to reaching the collector, thereby resulting in ribbon like flat nanofiber morphologies or conglutination of nanofibers at boundaries $[74,84]$. Phase separation occurs at the liquid-air interface when the jet is travelling through the atmosphere towards the collector, which is a function of the volatility of the solvent and influences the porosity of the nanofibers that are formed [74]. To achieve the desired porosity and nanofiber topographies a blend of two solvents (co-solvents) with different boiling points can be used. Table 1 lists the properties of key solvents used for electrospinning and their effect on nanofiber morphology.

Megelski and coworkers [79] were able to demonstrate the influence of solvents on the porosity of electrospun polystyrene nanofibers. Two solvents were employed, tetrahydrofuran (THF) (highly volatile) and dimethylformamide (DMF) (less volatile), with different boiling points and vapour pressures to electrospin polymer nanofibers. Nanofibers that were electrospun from THF had very high 
TABLE 1: Solvent properties and the resulting fiber morphology.

\begin{tabular}{|c|c|c|c|c|}
\hline Solvent & $\begin{array}{c}\text { Boiling } \\
\text { point }\left({ }^{\circ} \mathrm{C}\right)\end{array}$ & Other properties & Fiber morphology & Ref. \\
\hline DCM & 39.8 & $\begin{array}{l}\text { Low dielectric constant, high surface } \\
\text { tension }\end{array}$ & Beaded, large diameter & {$[77,85]$} \\
\hline Chloroform & 61.2 & High intrinsic viscosity & $\begin{array}{l}\text { Beaded at very low polymer concentration, smooth } \\
\text { at higher concentration }\end{array}$ & {$[86]$} \\
\hline Methanol & 64.7 & High dielectric constant & $\begin{array}{l}\text { Small fiber diameter with } \uparrow \text { methanol concentration } \\
\text { until } 50 \% \text { then } \uparrow \text { fiber diameter }\end{array}$ & {$[77]$} \\
\hline THF & 66 & High dipole moment, good conductivity & Smooth and beaded, ribbon-like, high pore density & {$[78,87]$} \\
\hline Ethyl acetate & 77.1 & High dielectric constant, fair conductivity & Smooth and beaded, ribbon-like & {$[87]$} \\
\hline Ethanol & 78.3 & $\begin{array}{l}\text { Low surface tension, high intrinsic } \\
\text { viscosity }\end{array}$ & Smooth, large diameter & $\begin{array}{c}{[83,85,} \\
86]\end{array}$ \\
\hline MEK & 79.6 & High dipole moment, good conductivity & Flat, ribbon-like, very few beads & {$[87]$} \\
\hline Dichloroethane & 83.5 & High dipole moment, fair conductivity & Smooth and beaded, C-shaped & {$[87]$} \\
\hline Water & 100 & Low intrinsic viscosity & Beaded, small diameter & {$[83,86]$} \\
\hline DMF & 153 & $\begin{array}{l}\text { High dipole moment, high conductivity, } \\
\text { low intrinsic viscosity }\end{array}$ & Smooth and beaded, round & {$[86,87]$} \\
\hline
\end{tabular}

(DCM: dichloromethane; THF: tetrahydrofuran; MEK: methyl ethyl ketone; DMF: dimethylformamide).

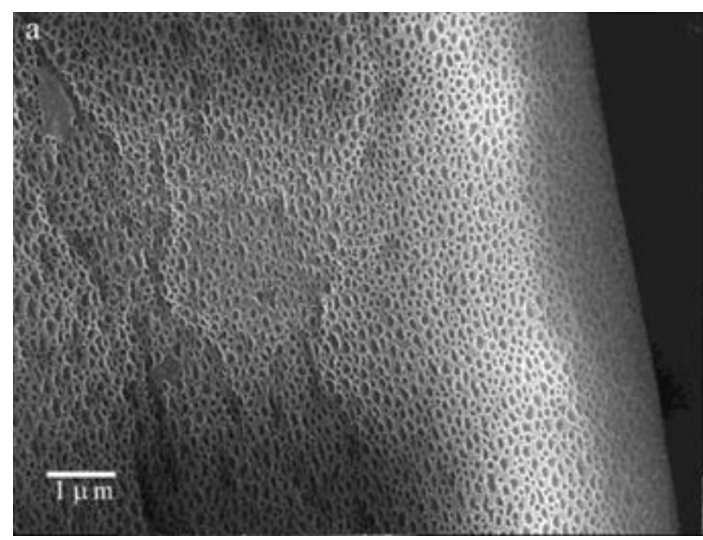

(a)

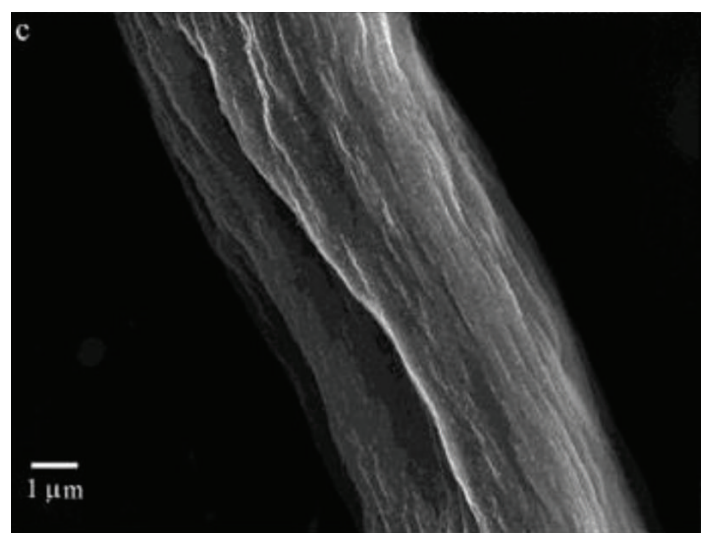

(c)

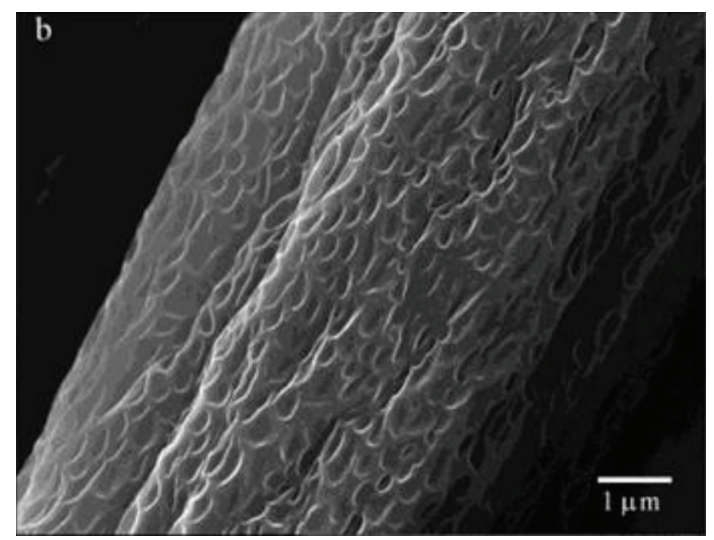

(b)

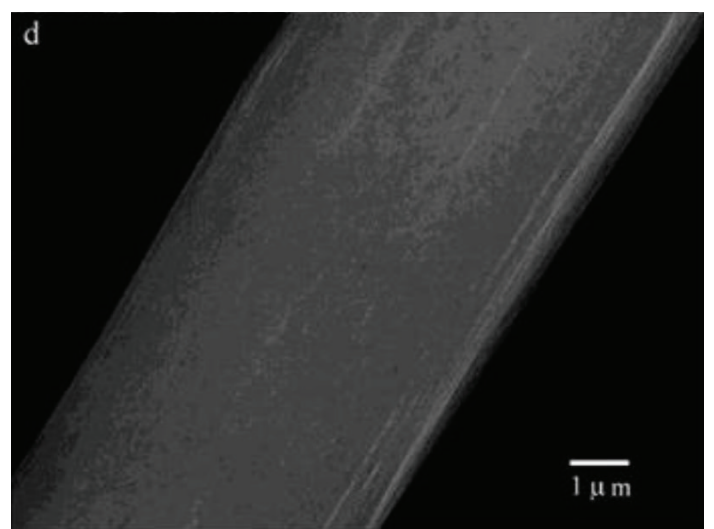

(d)

FIGURE 3: FESEM images of polystyrene fibers produced from polystyrene solutions of varying volatility: (a) 100\% THF; (b) 75\% THF/25\% DMF; (c) 50\% THF/50\% DMF; (d) 100\% DMF. Adapted from Megelski et al. [79]. 
surface pore density whereas nanofibers from DMF displayed almost completely smooth surface morphology. When a combination of the two solvents was used in varying ratios, the pore density decreased with an increase in the ratio of the less volatile solvent DMF (Figure 3). Jarusuwannapoom and coworkers [87] extensively investigated the effects of using different solvents on the electrospinning of polystyrene solutions. Eighteen different solvents were tested and it was found that only five (DMF, THF, ethyl acetate, 1,2dichloroethane, and methyl ethyl ketone) were optimal for electrospinning of polystyrene. All solvents had a high dipole moment value, displayed relatively superior conductivity and the polystyrene solutions had desirable conductivity values that are important factors for electrospinability.

Fong and coworkers [83] produced PEO nanofibers employing a mixture of ethanol and water as a co-solvent. By increasing the ratio of ethanol and maintaining the PEO concentration constant the solution viscosity increased, resulting in the formation of smooth nanofibers with larger diameters as depicted in Figure 4. The authors attributed this observation to the fact that ethanol was more volatile and therefore evaporated faster when a blend of solvents was used, resulting in a greater viscosity and facilitating nanofiber solidification with reduced bead formation. Meechaisue and coworkers [77] investigated the use of methanol, dichloromethane (DCM), and a combination of the two solvents to electrospin poly(DTE-co-20\%DT carbonate) nanofibers. Employing DCM (the more volatile of the two) as a solvent resulted in the formation of beaded nanofibers, while the combination of DCM and methanol produced smooth nanofibers. The authors suggested that the low dielectric constant and higher surface tension of DCM are responsible for the bead formation upon use of pure DCM as a solvent. Authors further reported that when the methanol content in the solvent increased from 10 to $30 \%$, the diameter of the nanofibers decreased, which they attributed to the higher dielectric constant of methanol, which resulted in greater charge repulsion within the nanofiber jet. However, when $50 \%$ of the solvent was methanol, the nanofiber diameter increased due to an increase in the electrostatic force, causing the nanofiber to reach the collector rapidly.

Son and coworkers [86] examined the influence of employing water, chloroform, DMF, and ethanol as solvents for electrospinning of PEO nanofibers. They observed that using DMF as a solvent required a higher applied voltage and a longer capillary-to-collector distance for electrospinning, in comparison to the other three solvents, which could rationally be attributed to the higher boiling point of DMF. The minimum polymer concentrations for the formation of beadfree nanofibers were 3, 4, 7, and 10\% W/V for chloroform, ethanol, DMF, and water, respectively. Employing water as the solvent resulted in the production of nanofibers with the smallest diameter in relation to the other three solvents. In another study by Yang and coworkers [85], the ability of electrospinning poly(vinyl pyrrolidone) (PVP) was investigated using different solvents. The solvents investigated were DCM, ethanol, and DMF. The use of ethanol produced smooth PVP nanofibers, whereas beaded nanofibers were formed from DCM and DMF solutions of PVP. Nanofibers electrospun from a combination of ethanol and DMF had small diameters of $20 \mathrm{~nm}$ whereas a combination of ethanol and DCM resulted in the formation of nanofibers with diameters as large as $300 \mathrm{~nm}$. It could therefore be concluded that nanofiber morphology and porosity may be regulated by the justified use of solvents or a combination of solvents.

\subsection{Effect of Solution Conductivity. The charge carrying} capacity of polymer solutions with high conductivity is greater than those with low conductivity and therefore the fiber jet produced from a solution of high conductivity is subjected to a greater tensile force when exposed to an applied voltage. Generally it has been observed that an increase in solution conductivity results in a substantial decrease in nanofiber diameter and it has been shown that the radius of the nanofiber jet is inversely related to the cube root of the electrical conductivity of the solution $[14,78,83,88]$. Fong and coworkers [83] investigated the influence of adding sodium chloride $(\mathrm{NaCl})$ to a solution of $\mathrm{PEO}$ in distilled water for electrospun nanofiber fabrication. The authors recorded an increase in the net charge density of the nanofiber jet upon addition of $\mathrm{NaCl}$ to the solution which resulted in a decrease in resistivity of the nanofiber jet formation due to the increased quantity of charge flowing into the jet. The increased charge density imparted an increased elastic force within the jet and uniformity to the nanofibers resulting in the formation of smooth nanofibers. When the charge of the fiber jet was neutralised with ions of opposite polarity, electrical forces were reduced and bead defects were observed [83].

Zong and coworkers [78] conducted a study to investigate the effect of adding different salts to PDLLA solutions for electrospinning. $\mathrm{KH}_{2} \mathrm{PO}_{4}, \mathrm{NaH}_{2} \mathrm{PO}_{4}$, and $\mathrm{NaCl}$ were the investigated and each was added separately at $1 \% \mathrm{~W} / \mathrm{V}$ to PDLLA solutions. The resulting electrospun nanofibers were smooth, bead-free and had smaller diameters than nanofibers electrospun from solutions that did not contain a salt. Solutions containing $\mathrm{NaCl}$ produced nanofibers with the smallest diameter while $\mathrm{KH}_{2} \mathrm{PO}_{4}$-containing solutions produced nanofibers with the largest diameter. The size of the ions was also found to have an effect on the nanofiber diameter. Ions with smaller radii have a higher charge density and impose greater forces of elongation on electrospun nanofibers. The addition of $\mathrm{NaCl}$ therefore produced nanofibers with the smallest diameter due to the fact that $\mathrm{Na}^{+}$and $\mathrm{Cl}^{-}$ions are smaller than potassium or phosphate ions. Son and coworkers [86] investigated the influence of adding small quantities of polyelectrolytes, poly(allylamine hydrochloride) (PAH), and poly(acrylic acid) (PAA) on the conductivity of PEO in water. The viscosity and surface tension of the formed solution increased only slightly, whereas conductivity and charge density increased significantly when small quantities of PAH and PAA were added. The decrease in resulting electrospun nanofiber diameters on addition of the polyelectrolytes was therefore attributed to the increase in solution conductivity.

Surfactants are often added to the solution to improve the conductivity or for stabilizing emulsion formation [89, 90]. Zeng and coworkers [91] examined the influence of 


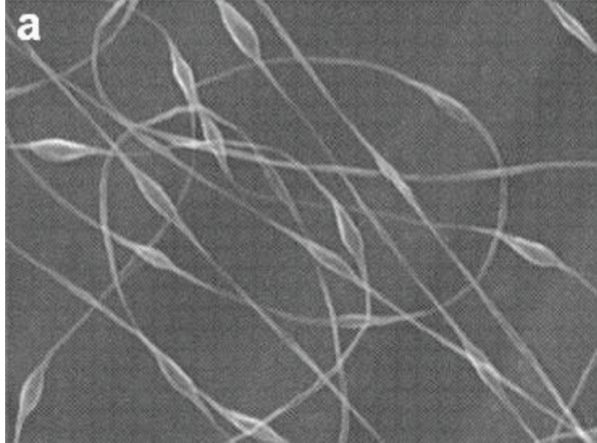

(a)

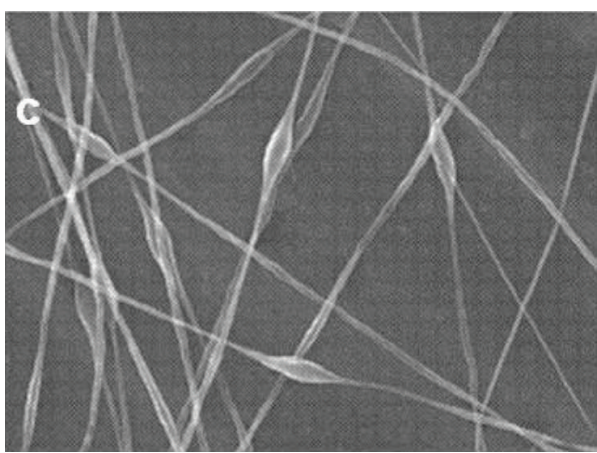

(c)

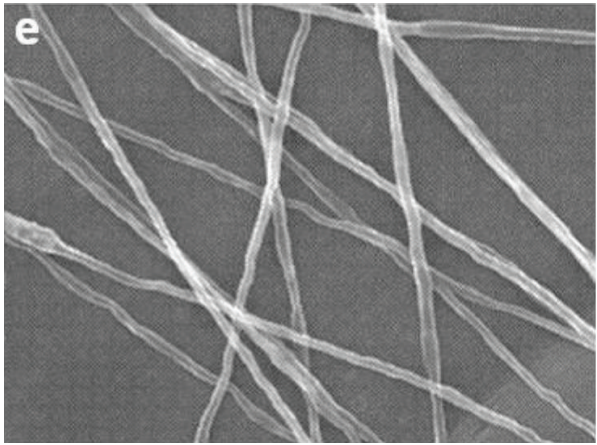

(e)

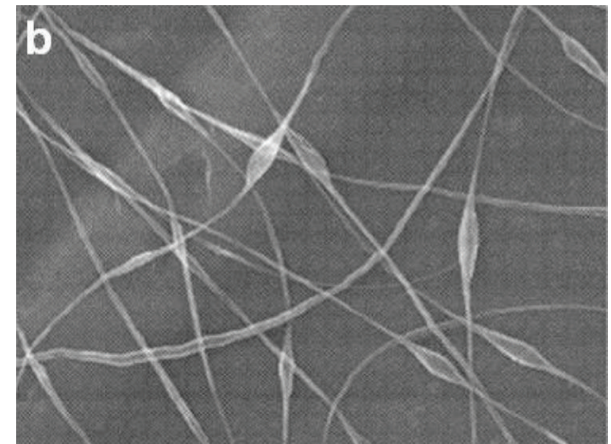

(b)

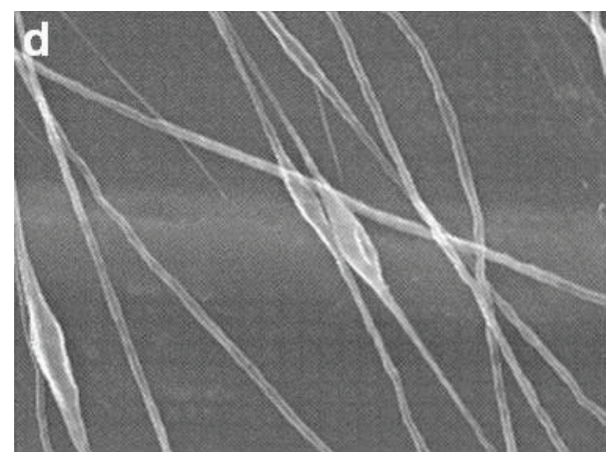

(d)

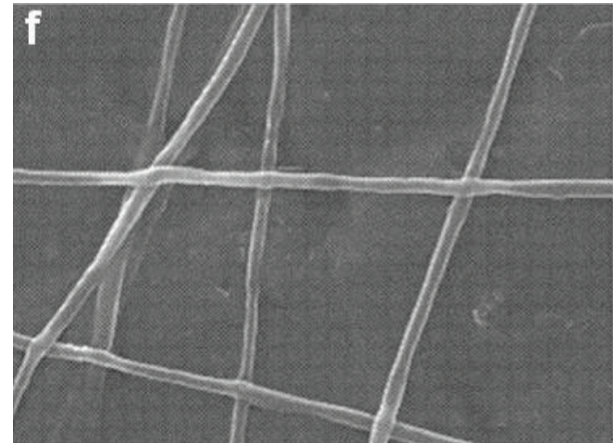

(f)

FIGURE 4: Varying PEO fiber morphology from beaded to smooth fibers as the ratio of ethanol is increased: (a) ethanol/water ratio $=0.000$; (b) ethanol/water ratio $=0.054 ;(c)$ ethanol $/$ water ratio $=0.115$; (d) ethanol $/$ water ratio $=0.260 ;(\mathrm{e})$ ethanol $/$ water ratio $=0.448$; and $(\mathrm{f})$ ethanol/water ratio $=0.702$. Adapted from Fong et al. [83].

surfactants and drugs on the uniformity and diameter of electrospun poly(L-lactic acid) (PLLA) nanofibers. The surfactants employed were triethyl benzyl ammonium chloride (TEBAC), sodium dodecyl sulphate (SDS), and aliphatic PPO-PEO ether (AEO10). Reduction in nanofiber diameter and improvement of uniformity was observed with the addition of all three types of surfactants. The solution conductivity is determined by the constituent polymer, solvent, and ionisable salts, the combination of which can impact the nanofiber size by $1-2$ orders of magnitude [74].

2.6. Effect of the Capillary and Collector Distance. The capillary to collector distance influences the size and morphology of the nanofibers formed. However, its effect is relatively less profound compared to the other processing variables discussed. An optimum distance between the capillary and collector is desirable for nanofiber formation and on either side of this range bead formation or electrospraying instead of electrospinning may be observed [23]. Generally, with an increase in the distance between the capillary and the collector the diameter of electrospun nanofibers decreases [15]. At smaller distances the solvent does not have sufficient time to evaporate completely resulting in nanofibers with flattened structures due to inadequate drying. In a study conducted by Baumgarten [14], the effect of increasing the distance between the capillary and the collector on the nanofiber diameter (when the applied voltage was increased) was investigated. At a distance of $50 \mathrm{~mm}$ between the capillary and collector, the nanofiber diameter initially decreased to a minimum and 
then increased with increasing voltage. At a gap of $75 \mathrm{~mm}$, the decrease in nanofiber diameter to a minimum was not apparent and the diameter increased slightly in response to an increase in the applied voltage. Doshi and Reneker [15] reported a decrease in nanofiber diameter with an increase in the distance up to $30 \mathrm{~mm}$, beyond which the fiber jet became too small and unstable. Megelski and coworkers [79] noted no significant change in nanofiber diameter with a change in distance between the capillary and the collector. However, they reported an initiation of bead formation as the distance decreased.

\section{The Application of Electrospun Nanofibers in Drug Delivery Systems Design}

Electrospinning has gained much attention in fabricating nanofibers that have found application as drug carriers for drug delivery systems. There are two main features of electrospun nanofibers (configured as nanomats) which make them attractive as drug carriers. Firstly, the already large surface area to volume ratio of nanofibers is dramatically increased when considering the porosity of the electrospun nanofibers. Larger surface areas overcome the limitation of high drug uptake which is generally associated with other conventional systems. In addition, it overcomes the constraint of drug diffusion due to the high surface area and porous interconnected architecture, leading to an increase in the total fraction of drug released. Secondly, the control and "tailoring" of the matrix properties, such as the diameter, porosity, and morphology by varying the processing variables and type of materials can regulate the drug release profile. A number of drugs such as antibiotics [92, 93], anticancer agents [94, 95], anti-inflammatory agents [54], proteins [96], and DNA $[55,97]$ have been incorporated into electrospun mats and delivered to desired targets in the body. Nanofibrous carriers can also offer site-specific delivery of more than one drug into the body [98].

Many drug-loaded electrospun nanofibers have been reported as drug delivery systems and a few in vivo experiments undertaken are related to cancer research. Drug delivery implants that provide site-specific sustained release of an anticancer drug at a tumour have been produced by $\mathrm{Xu}$ and coworkers [94] as well as Ranganath and Wang [95]. Another example is the site-specific delivery of heparin to the site of a vascular graft using electrospun nanofibers [99]. Electrospun fibrous mats have also been investigated for their application in transdermal drug delivery systems or as wound dressings [5, 54, 100-103]. The prevention of postsurgical abdominal adhesions and infection has been explored using antibiotic-loaded electrospun fibrous scaffolds [57, 93].

\subsection{Polymers Employed for Electrospinning in Drug Delivery.} Controlled site-specific drug delivery over a period of time is achievable by employing drug delivery matrices composed either of biodegradable or nondegradable polymers. Depending on the polymer type selected the mechanism of drug release may only be via diffusion in the case of nondegradable polymers and, by matrix erosion (in addition to diffusion) for biodegradable polymers. The drug delivery rate can thus be modulated by the choice of polymer(s) for an electrospun matrix. Various polymers have been electrospun and applied in drug delivery as shown in Table 2. The polymer type, solvent, and drug compatibility are important processing variables when attempting to achieve stable and reproducible drug delivery. As a general rule, lipophilic polymers should be used with lipophilic drugs while hydrophilic polymers should be used with hydrophilic drugs in order to obtain a stable release profile [104].

3.2. Drug Loading and Drug Release from Electrospun Nanofibers. Loading of drug into electrospun nanofibrous matrices has been reported by using various methods, such as, coating, embedding, or encapsulating in order to achieve control over the drug release kinetics [54, 95, 96, 99, 110]. If the drug and polymer are soluble in the same solvent, the drug can be dissolved directly into the polymer solution $[54,95,99]$, or in the case where the drug and polymer are not soluble in the same solvent, the drug can be solubilized in a small quantity of another solvent before being added to the polymer solution $[3,53,99]$. Electrospinning of such solutions results in drug being embedded in the fabricated nanofiber scaffold. In another approach for drug and polymer insolubility in a common solvent, the drug can be dissolved in a solvent that is immiscible with that in which the polymer is dissolved and the two solutions can be loaded in separate capillaries in order to be electrospun coaxially $[92,100]$, or the two solutions could be blended, resulting in an emulsion that can be electrospun $[108,111]$. This approach leads to the encapsulation of the drug in the polymeric matrix. There is yet another technique for loading the drug after electrospinning. In this method drug is absorbed into the electrospun nanofibers by immersing the nanofibers in a drug solution [109].

The release of the drug from nanofiber matrices is mainly by the mechanisms of: desorption from the nanofiber surface, diffusion through the channels and pores of nanofibers or matrix degradation [74]. The drug release kinetics can be modulated by the choice of polymer and through control over the nanofiber diameter, porosity, geometry, and morphology by adjusting the various processing variables during electrospinning.

3.2.1. Effect of Dissolving Drug in the Polymer Solution. Ranganath and Wang [95] fabricated paclitaxel-loaded poly(D,Llactide-co-glycolide) (PLGA) copolymeric microfiber and submicrofiber implants by electrospinning PLGA 85:15 and PLGA 50:50 copolymers, respectively. PLGA was dissolved in a mixture of DCM and DMF at $30 \% \mathrm{~W} / \mathrm{V}$ and the drug paclitaxel was added to the polymer solution and the resulting solution was electrospun. Nanofibers were collected as mats on a rotating drum covered with aluminium foil. The mats were cut into sheets or punched as discs. Paclitaxel encapsulation efficiency was found to be $98 \pm 4.9 \%$ for PLGA $85: 15$ copolymeric microfibers and $94 \pm 0.57 \%$ for PLGA $50: 50$ copolymeric nanofibers. The developed implants were investigated against malignant glioma in vitro and in vivo. 
TABLE 2: Polymers, drugs, and solvent types for application in drug delivery.

\begin{tabular}{|c|c|c|c|}
\hline Polymer & Solvent & $\operatorname{Drug}(\mathrm{s})$ & Ref. \\
\hline \multirow{3}{*}{ Cellulose acetate } & \multirow{3}{*}{$2: 1$ acetone/DMAc } & Naproxen, indomethacin, ibuprofen, sulindac & {$[54]$} \\
\hline & & Curcumin & {$[103]$} \\
\hline & & Vitamin $\mathrm{A}$ and $\mathrm{E}$ & {$[102]$} \\
\hline & $7: 3 \mathrm{DCM} / \mathrm{methanol}$ & Heparin & [99] \\
\hline PCL & $3: 1$ chloroform/ethanol & Resveratrol, gentamycin & {$[92]$} \\
\hline PEO/PCL blend & Chloroform & Lysozyme & {$[3]$} \\
\hline \multirow{2}{*}{ PVA } & \multirow{2}{*}{ Deionised water } & Ketoprofen & {$[53]$} \\
\hline & & $\begin{array}{l}\text { Sodium salicylate, diclofenac, naproxen, } \\
\text { indomethacin }\end{array}$ & {$[101]$} \\
\hline Gelatin/PVA blend & Gelatin in formic acid, PVA in deionised water & Raspberry ketone & {$[105]$} \\
\hline PLGA & $\begin{array}{l}\mathrm{DCM} / \mathrm{DMF} \\
\mathrm{DMF}\end{array}$ & $\begin{array}{c}\text { Paclitaxel } \\
\text { Cefoxitin sodium }\end{array}$ & $\begin{array}{l}{[95]} \\
{[93]}\end{array}$ \\
\hline Polyurethane & $\begin{array}{l}\text { DMF } \\
\text { DMAc }\end{array}$ & $\begin{array}{l}\text { Itraconazole } \\
\text { Ketanserin }\end{array}$ & {$[5]$} \\
\hline \multirow{3}{*}{ PLLA } & $2: 1$ chloroform/acetone & $\begin{array}{l}\text { Doxorubicin } \mathrm{HCl} \\
\text { Tetracycline } \mathrm{HCl}\end{array}$ & $\begin{array}{l}{[104]} \\
{[100]}\end{array}$ \\
\hline & Chloroform & Cytochrome C & {$[4]$} \\
\hline & DCM & Bovine serum albumin & {$[106]$} \\
\hline PEVA & Chloroform & Tetracycline $\mathrm{HCl}$ & {$[107]$} \\
\hline PEG-PLA & Chloroform & Doxorubicin $\mathrm{HCl}$ & {$[94,108]$} \\
\hline PAA/PAH blend & Deionised water & Methylene blue & [109] \\
\hline
\end{tabular}

PCL: poly(e-caprolactone); PEO: poly(ethylene oxide); PVA: poly(vinyl alcohol); PLGA: poly(D,L-lactide-co-glycolide); PLLA: poly(L-lactic acid); PEVA: poly(ethylene-co-vinyl acetate); PEG: poly(ethylene glycol); PAA: poly(acrylic acid); PAH: poly(allylamine hydrochloride).

A sustained release of paclitaxel was observed over a period of 80 days in vitro and there was a low initial burst release recorded. The release of drug was nearly zero-order for the discs. The authors reported higher release from the nanofibers compared to microfibers which they attributed to the high surface to volume ratio and higher degradation rate of the nanofibers. The system was able to inhibit tumour growth of $69-78 \%$ for the microfibers and micro-disc treated animal groups after a tumour growth of 24 days.

Tungprapa and coworkers [54] prepared drug-loaded electrospun cellulose acetate nanofibrous mats for investigation as topical drug delivery carriers. Cellulose acetate $(16 \% \mathrm{~W} / \mathrm{V})$ was dissolved in a mixture of acetone and $N$, $\mathrm{N}$-dimethylacetamide (DMAc). The model drugs were four different nonsteroidal anti-inflammatory drugs (NSAIDs), that is, naproxen, indomethacin, ibuprofen, and sulindac. The drugs ( $20 \mathrm{wt} \%$ of polymer) were added individually to the cellulose acetate solutions and the resulting solutions were electrospun. The resulting cellulose acetate nanofiber mats had smooth morphology with no drug aggregates on the surface, indicating complete drug entrapment in the nanofibrous mats. The drug encapsulation efficiency of the nanofibrous mats was reported as $84-93 \%$. The drug release characteristics of the electrospun nanofiber mats as determined by the total immersion method in acetate buffer at $37^{\circ} \mathrm{C}$ was observed to be greater than the corresponding as-cast films. The maximum release of drug, as recorded from different drug-loaded electrospun cellulose acetate nanofiber mats in order of their ranking was naproxen followed by ibuprofen, indomethacin, and lastly sulindac.

Suwantong and coworkers [103] similarly electrospun curcumin-loaded cellulose acetate nanofibrous mats by using curcumin at $5,10,15$, and $20 \% \mathrm{~W} / \mathrm{V}$. Based on the cellulose acetate content, the drug encapsulation efficiency was recorded as $101.9 \pm 0.8 \%, 95.6 \pm 2.5 \%, 91.4 \pm 0.4 \%$, and $90.8 \pm$ $0.4 \%$ for the $5,10,15$, and $20 \% \mathrm{~W} / \mathrm{V}$ solutions, respectively. An initial burst release of curcumin was observed followed by a gradual increase in curcumin release over 50 hours. The maximum curcumin release was reported to be greatest for the $20 \% \mathrm{~W} / \mathrm{V}$ solution and lowest for the $5 \% \mathrm{~W} / \mathrm{V}$ solution.

Taepaiboon and coworkers [101] incorporated four different NSAIDs into electrospun PVA nanofibrous mats for transdermal drug delivery. PVA was dissolved in distilled water and the model drugs, sodium salicylate, diclofenac sodium, naproxen, and indomethacin, were added individually to the PVA solutions. These solutions were electrospun and the nanofibers were collected on a rotating metal drum. Drug encapsulation efficiency of electrospun PVA nanofibers ranged from 81 to $98 \%$. A burst release of sodium salicylate was exhibited by the nanofibrous mats on account of the high water solubility of the drug. As the nanofibrous matrix absorbed water and swelled, the drug solvated and rapidly 
leached out of the matrix. The total percentage of drug release from the nanofibrous mats at 24 hours was $98,97,76$, and $42 \%$ for naproxen, sodium salicylate, diclofenac sodium, and indomethacin, respectively. The same group of researchers further investigated the potential of electrospinning cellulose acetate solution loaded with vitamins A (all-trans retinol acid) and vitamin $\mathrm{E}$ ( $\alpha$-tocopherol) [102]. Solutions were prepared by dissolving $17 \% \mathrm{~W} / \mathrm{V}$ cellulose acetate and vitamin $\mathrm{A}$ or vitamin $\mathrm{E}$ in a mixture of acetone and DMAc and were electrospun and nanofibers were investigated for in vitro drug release characteristics. Drug encapsulation efficiency of $82.9 \pm 2.2 \%$ and $44.5 \pm 1.1 \%$ was achieved for vitamins $E$ and $\mathrm{A}$, respectively, and a gradual increase in the rate of release was recorded for both the vitamins from the nanofibrous mats in comparison to the burst release from comparative as-cast films. A maximum release of approximately $52 \%$ and $34 \%$ was recorded for vitamin $\mathrm{E}$ and vitamin $\mathrm{A}$, respectively, and this was achieved after a time period of 24 hours and 6 hours, respectively.

Verreck and coworkers [5] investigated the possibility of incorporating water soluble drugs into hydrophobic polyurethane (PU) electrospun nanofibers for application in topical drug administration. Solutions were made by dissolving PU and itraconazole in DMF as well as PU and ketanserin in DMAc. The release of itraconazole from electrospun PU nanofibers increased gradually over 20 hours and there was no initial burst release. Ketanserin release was observed to be faster than itraconazole during the first 4 hours, after which, ketanserin released slowly over 24 hours.

Zeng and coworkers [104] investigated the influence of the compatibility between the polymer solution and drug on the release kinetics of electrospun nanofiber formulations. Paclitaxel, doxorubicin $\mathrm{HCl}$, and doxorubicin-base were employed as the model drugs. PLLA was dissolved in a mixture of chloroform and acetone and the drugs were added to the solutions separately. Paclitaxel and doxorubicin-base showed superior compatibility with PLLA and desirable solubility in the chloroform/acetone co-solvent system that was used, which resulted in optimum drug encapsulation. The authors observed inadequate encapsulation of doxorubicin $\mathrm{HCl}$ into the nanofiber matrix and the drug was confined near or on the surface of the PLLA nanofibers. The release rate of paclitaxel and doxorubicin-base was nearly zero-order due to nanofiber degradation whereas, a burst release of doxorubicin $\mathrm{HCl}$ was observed due to fast desorption from the nanofiber surface. It was concluded that drug solubility and compatibility with solvents and polymers are important factors to be considered for adequate entrapment of drug molecules in electrospun nanofiber matrices.

3.2.2. Effect of Solubilizing Drug Prior to the Addition of the Polymer Solution. Kenawy and coworkers [107] reported the applicability of electrospun nanofiber mats as drug delivery systems using poly(ethylene-co-vinyl acetate) (PEVA), poly(lactic acid) (PLA) and a 50:50 blend of the two polymers. The polymers were individually dissolved in chloroform and tetracycline $\mathrm{HCl}$, which was used as a model drug, and thereafter added to the polymer solutions. The drug release rate as recorded from individual polymeric nanofiber mats was highest for PEVA, releasing approximately 65\% of drug followed by 50:50 PLA/PEVA blended nanofiber mats that displayed a release of $50 \%$ drug content over a period of 5 days. PLA nanofiber mats exhibited an initial burst release effect arising from desorption of drug adhering to the surface of nanofibers followed by a negligible release over the next 50 hours. Authors compared the release profile of electrospun mats with corresponding films and reported greater release from the former which they explained on the basis of higher surface to volume ratio of the electrospun mats. The same group of authors developed another system of controlled drug release by electrospinning partially and fully hydrolysed PVA from deionised water [53]. The drug, ketoprofen dissolved in a small amount of methanol, was added to the polymer solution. It was observed that fully hydrolysed PVA with entrapped ketoprofen could only be electrospun when combined with a small amount of surfactant (Triton X-100) and acetic acid. Additionally, authors stabilized the drug embedded PVA matrix with methanol treatment which resulted in crosslinking and eliminating the burst release of the drug from fiber matrix. Furthermore, methanol stabilized matrix exhibited a slow drug release over a period of two weeks and also, the overall drug release was lower from treated fibers. Authors reported the degree of hydrolysis of PVA to have additional effect on the drug release rate.

Kim and coworkers [93] investigated the release of hydrophilic drug, cefoxitin sodium, from hydrophobic PLGA-based electrospun fibrous scaffolds. PLGA was dissolved in DMF at 33\% W/V. Cefoxitin sodium was dissolved in a small amount of water and this aqueous drug solution was slowly added to the polymer solution and the obtained drug/polymer solution was electrospun. The drug concentration was found to influence the morphology and density of the scaffolds. As the drug concentration increased, the scaffold density decreased and the fiber morphology changed from bead-and-string to fibers due to the salt effect during electrospinning. Adding amphiphilic poly(ethylene glycol) PEG-b-PLA block copolymer to the electrospinning solution resulted in fiber scaffold exhibiting sustained release of the drug up to one week. Addition of amphiphilic polymer accounted for drug being embedded in the polymer matrix which was earlier located at the fiber surface due to less physical interaction with hydrophobic polymer and higher ionic strength of hydrophilic drug. In another study, Kenawy and coworkers [53] examined the controlled release of a protein drug from electrospun fiber meshes composed of a blend of poly( $\varepsilon$-caprolactone) (PCL) and PEO. Lysozyme was employed as a model protein drug. A blend of PCL and $\mathrm{PEO}$ was dissolved in chloroform at varying ratios. Salt-free and dried lysozyme was dissolved in dimethylsulphoxide and the resulting solution was mixed with the polymer blend solution. Protein release studies from electrospun polymeric mesh were carried out and it was found that blends containing a large amount of PEO demonstrated a more rapid release of the model protein. Lysozyme release thus could readily be controlled by varying the polymer blend ratio. In a study by Luong-Van and coworkers [99], heparin-loaded nanofibrous PCL mats were fabricated by electrospinning. Heparin was 
dissolved in a small quantity of water and methanol was added. This solution was added to DCM and the resulting solution was used to dissolve PCL. The polymer/drug solution was electrospun and the nanofibers were collected on glass microscope slides. The authors reported that heparin was released from the nanofibrous mats in a sustained manner over a period of 14 days.

\subsubsection{Coaxial Electrospinning. Coaxial electrospinning} allows for electrospinning two different polymer solutions or a polymer solution and a drug reservoir in a single setup while loaded as separate entities in respective concentric capillaries. This mode of electrospinning form of core-shell nanofibers is especially useful in fabricating nanofibers that encapsulate the drug within the core thereby minimizing initial burst release of the drug and shifting the release mechanism mainly to drug diffusion or nanofiber degradation rather than desorption from nanofiber surface. Drug-loading and release in such systems can be regulated by optimizing the feed-rate of drug solution, higher feed-rates provide higher drug-loading and visa-versa. A schematic for coaxial electrospinning apparatus is shown in Figure 5 [112].

Huang and coworkers [92] fabricated drug-loaded double-layered PCL nanofibers by coaxial electrospinning. PCL was dissolved in a mixture of chloroform and ethanol and the model drugs, resveratrol, and gentamycin sulphate were dissolved in ethanol and water, respectively. The polymer concentration was kept constant and the drug concentrations varied. The drug and polymer solutions were fed through coaxial needles, with the drug solution in the inner core needle and the polymer solution in the outer needle. A potential was applied and nanofibers, with a drug core and an outer polymer sheath, were collected on a grounded metal screen. It was found that when the drug and polymer solutions were miscible (resveratrol-loaded nanofibers), an increase in drug concentration resulted in a decrease in bead defects, but when the drug and polymer solutions were immiscible (gentamycin sulphate loaded nanofibers), the opposite was true. The drug release profile of PCL nanofibrous mat exhibited no burst release, instead a consistent release was observed for both drugs over-sevenday a period. This release of encapsulated drug is attributed to the pace of PCL matrix degradation.

In a study by $\mathrm{He}$ and coworkers [100], drug-loaded coreshell nanofibers of PLLA were produced by coaxial electrospinning. PLLA was dissolved in a mixture of chloroform and acetone and the model drug; tetracycline $\mathrm{HCl}$ was dissolved in a mixture of methanol and chloroform with a small amount of PLLA. Triallyl isocyanurate was added to the polymer solution as a cross-linking agent. Drug concentration was kept constant while polymer concentration was varied. The drug solution was fed through the inner needle and the polymer solution through the outer needle and the fibers collected depicted sustained drug release over a period of 32 days (Figure 6). The authors observed that a lower polymer concentration resulted in the formation of more uniform nanofibers with narrow diameter distribution, whereas, higher polymer concentrations resulted in the formation of larger diameter nanofibers (Figure 7). The authors thus

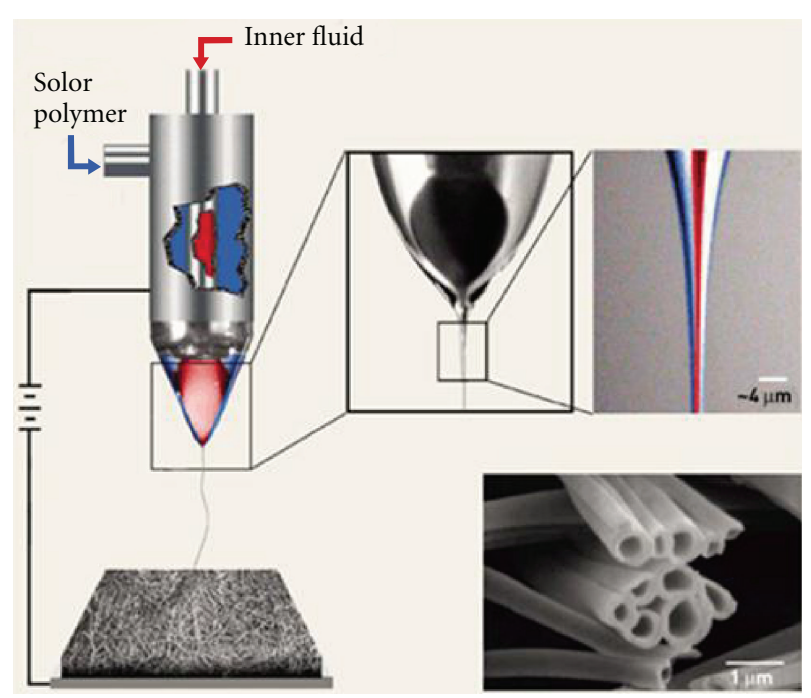

FIGURE 5: Schematic showing the coaxial electrospinning apparatus setup. Adapted from Jacoby [112].

inferred that drug release rate may be adjusted by changing the polymer concentration and hence shell thickness.

Wang and coworkers [113] prepared biodegradable coreshell nanofibers by coaxial electrospinning of PDLLA and poly(3-hydroxybutyrate) (PHB) solution. They prepared PDLLA/PHB or PHB/PDLLA core-shell nanofibers by reversing the polymer solutions in the inner and outer capillaries. Authors reported the flow-rate of core liquid to have predominant effect on both outer and inner fiber diameter. The authors have extensively surveyed the effect of flow-rate on morphologies of the tailor cone, the polymer jet, and the electrospun nanofibers. The two polymers PDLLA and PHB in fibrous mesh coexisted in amorphous and crystalline state, respectively. The PDLLA/PHB nanofibers were used for the delivery of the drug dimethyloxalylglycine (DMOG) - a proangiogenic compound-from the core section. The polymeric nanofibers exhibited two-stage drug release kinetics in contrast to the burst release from electrospun nanofibers of either PDLLA of PHB alone. Nanofibers exhibited 25\% drug release for the first stage (in 60 hours) followed by linear release that was controlled by the nanofiber thickness of the PHB shell of $70 \%$ of the drug.

3.2.4. Emulsion Electrospinning. Emulsion electrospinning was first proposed by $\mathrm{Xu}$ and coworkers [108] and the morphological details of nanofiber forming during emulsion electrospinning have been exemplified as shown in Figure 8 [114]. $\mathrm{Xu}$ and coworkers electrospun water soluble drug loaded ultrafine nanofibers of amphiphilic PEG-co-PLLA diblock copolymers from water-in-oil (W/O) emulsions [108]. Doxorubicin $\mathrm{HCl}$, as the model drug, was dissolved in water at varied concentrations to form the aqueous phase. Amphiphilic PEG-b-PLLA diblock copolymer was dissolved in chloroform with SDS as an emulsifying agent and the drug solution was slowly added to this polymer solution with stirring. The resulting $\mathrm{W} / \mathrm{O}$ emulsion was electrospun into fine nanofibers which exhibited complete drug encapsulation 


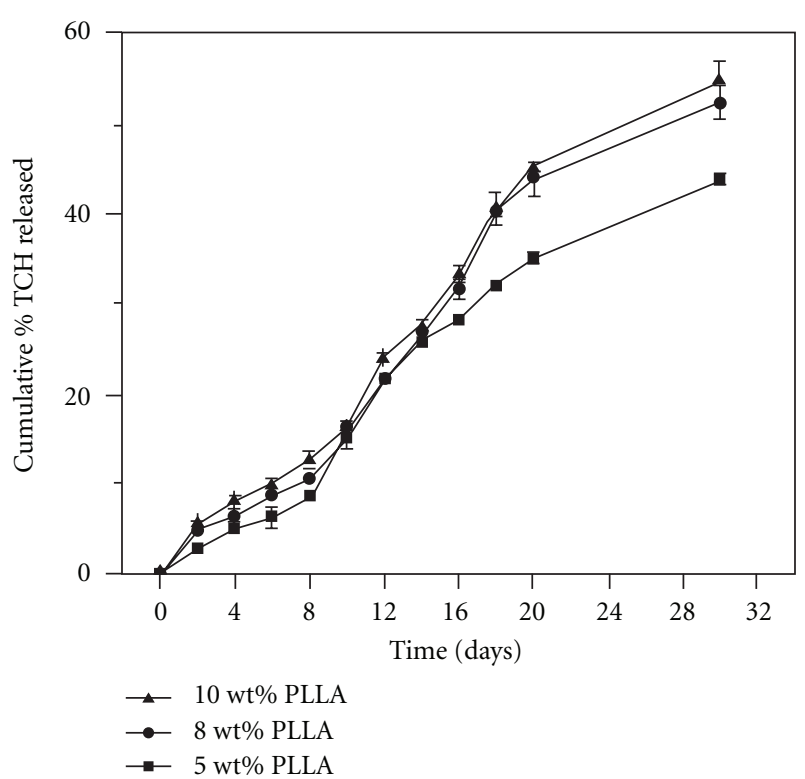

Figure 6: Sustained in vitro release of drug tetracycline hydrochloride from coaxially electrospun fibrous membranes with different PLLA shell concentrations (in tris buffer solution at $\mathrm{pH} 7.35,37^{\circ} \mathrm{C}$ ). Adapted from He et al. [100].

as the drug was soluble in the aqueous phase of a stable emulsion. The drug release from electrospun copolymeric nanofibers was controlled by diffusion and enzymatic degradation of the matrix and was reported to have occurred slowly over 10 hours.

Maretschek and coworkers [4] explored the loading of protein in hydrophobic PLLA nanofibers by electrospinning. Cytochrome C, taken as the hydrophilic model protein was dissolved in water and PLLA in chloroform. Hydrophilic polymers such as poly(ethylene imine) (PEI) or poly(l-lysine) (PLL) were added to the aqueous phase before emulsion preparation in order to reduce the hydrophobicity of the resulting fiber mesh. Protein encapsulation efficiency of electrospun PLLA was calculated to be between 85 and 95\%. PLLA nanofibers exhibited slow controlled release behaviour and authors anticipated the protein release to be dependent on the surface tension of the release medium as well. The concentration of polymer in electrospinning solution exhibited additional effect on drug release profile of corresponding polymeric matrix with higher PLLA concentrations exhibiting no burst release whereas lower concentrations resulting in an increased release rate. The authors reported that the incorporation of hydrophilic polymers into the aqueous phase resulted in a larger fiber diameter and an adjustable protein release rate.

Qi and coworkers [106] produced PLLA composite nanofibers by electrospinning from $\mathrm{W} / \mathrm{O}$ emulsions. Alginate was dissolved in water and bovine serum albumin (BSA) was taken as the model drug and added to the alginate solution in water. The aqueous solution was then slowly added to DCM comprising the surfactant sodium bis(2ethylhexyl) sulfosuccinate and the mixture was vortexed. A calcium chloride solution was added to the vortexed mixture in order to cross-link the alginate to form calcium alginate gel beads. PLLA was then added to the emulsion and dissolved in the DCM phase before being electrospun into nanofibers. The calcium alginate beads were spindle shaped upon electrospinning and served as drug reservoirs interspersed within the PLLA nanofiber mesh. The authors observed an initial burst release due to protein adsorbed or loosely associated on the fiber surface followed by a slow release over a period of 120 hours.

$\mathrm{Li}$ and coworkers [111] adopted emulsion electrospinning technique for loading proteinase $\mathrm{K}$ inside PEG-PLA nanofibers in a novel approach of preparing self-accelerated biodegradable membranes. Aqueous solution of proteinase $\mathrm{K}(0.02 \mathrm{mg} / \mathrm{L})$ was slowly added to the PEG-PLA solution $(0.08 \mathrm{~g} / \mathrm{mL})$ in chloroform with $1 \%$ SDS w/w, under magnetic stirring. The prepared emulsion had volume ratio of enzyme solution to polymer solution as $1: 25$. The formed nanofibers had a core-shell structure, with proteinase $\mathrm{K}$ forming the core and PEG-PLA forming the shell. The authors observed that the nanofiber core and shell diameters could be adjusted by changing the size of emulsion droplets before electrospinning. The hydrophilic PEG component in polymer offered affinity and compatibility for superior loading of the hydrophilic molecule proteinase $\mathrm{K}$. The enzymatic activity of proteinase $\mathrm{K}$ was retained after electrospinning as evident from the in vitro biodegradation studies which reported almost $100 \%$ mass loss of the mesh after 7 days with no significant mass loss reported in case of mesh devoid of proteinase $\mathrm{K}$.

3.2.5. Drug Loading by Absorption. Drug loading approaches discussed thus far involved the encapsulation of drug within the electrospun nanofiber mesh during the process of nanofiber fabrication, that is, drug was electrospun with the polymer to yield a drug-loaded nanofiber matrix. To avoid subjecting drug molecules to the electrostatic forces during electrospinning or to bypass elaborate preprocessing of drug via encapsulation before electrospinning, drug loading into the formed electrospun nanofiber mesh may be undertaken by immersing the mesh in a drug solution. This approach has been investigated by Chunder and coworkers [109] for loading drug in electrospun PAA/PAH nanofibers [109]. The authors strategically selected two oppositely charged polymers which are weak polyelectrolytes, as they intended to a design nanofiber mesh that was responsive to a change in environment. Methylene blue (cationic molecule) was employed as a model drug. Drug loading was achieved by immersing the crosslinked polymeric mesh in a nonbuffered solution of $1 \%$ methylene blue for 1 hour. The drug-loaded mesh was dried after rinsing off the loosely bound drug with an appropriate solution. Negatively charged carboxylate groups in the nanofiber hold the positively charged drug in the polymeric mesh and release it under controlled conditions. The degree of ionisation of PAA was dependent on the $\mathrm{pH}$ of the dissolving medium and drug release therefore could be controlled by changing $\mathrm{pH}$ of the medium. As the $\mathrm{pH}$ of the nonbuffered dissolving medium decreased below 6 , protons began to bind to carboxyl groups, resulting in the release of methylene blue into the medium. When the nanofibers 


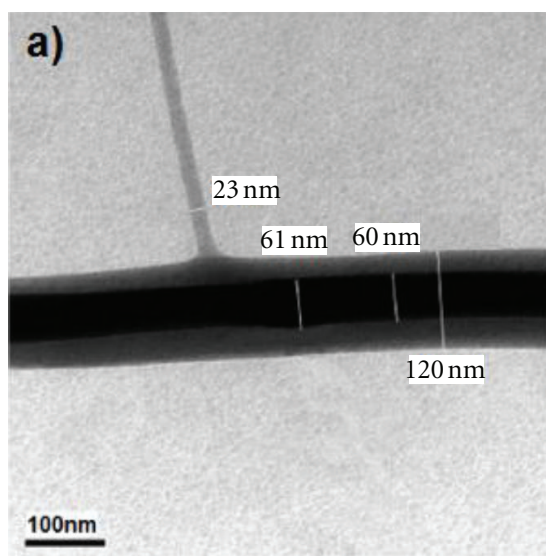

(a)

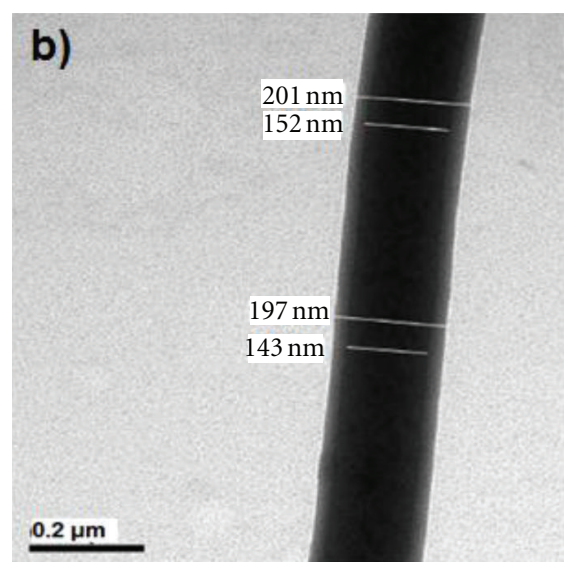

(b)

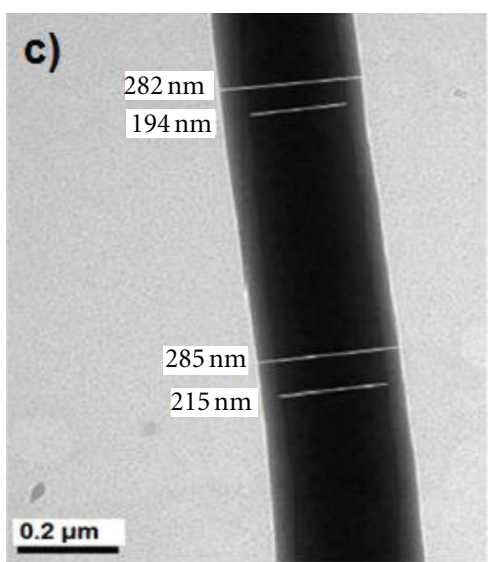

(c)

Figure 7: TEM images of the shell-core PLLA nanofibers with the $5 \mathrm{wt} \% \mathrm{TCH}$ (containing $1 \mathrm{wt} \%$ PLLA) in the core and (a) $5 \mathrm{wt} \%$, (b) 8 wt\%, and (c) $10 \mathrm{wt} \%$ PLLA in the shell. Adapted from He et al. [100].

were placed in phosphate buffered saline (PBS), methylene blue underwent a fast release due to swelling caused by the PBS. It was observed that the drug release could additionally be controlled by coating the nanofiber surfaces. Coating the nanofibers with a perfluorosilane network, that would not swell in PBS, resulted in obstruction of the diffusion of methylene blue and therefore achieving a sustained release in PBS. Furthermore the authors were able to achieve temperaturecontrolled release of methylene blue by coating the nanofibers with multilayers of PAA and poly(N-isopropylacrylamide) (PNIPAAM).

Bölgen and coworkers [115] achieved loading of the drug Biteral on electrospun PCL from chloroform/DMF solution by the same process. An electrospun PCL mesh was covered with a Biteral solution that resulted in drug absorption. The drug was found located on the surface of the nanofibers, which was responsible for the initial burst release of the drug accounting to almost $80 \%$ release in first 3 hours and complete release in above 18 hours. The authors justified the application of such a rapid initial burst release in postsurgery treatments where maximum infection occurs in initial few hours.

3.3. Cross-Linking of Fibers. Electrospun nanofiber meshes are promising candidates for fabrication of drug delivery systems especially because of the high surface area of the nanofibers. However, the electrospun nanofibers generally lack good mechanical and structural integrity especially when biodegradable or natural polymers form a part of the electrospun mesh. Crosslinking such matrices may provide for sustained and controlled drug release which otherwise would have been influenced by the fast structural loss or instability of the nanofiber mesh on exposure to release medium in vitro or the physiological environment in vivo. Figures 9(a) and 9(b) depict the loss in structural integrity of the AS-spun gelatine nanofiber mesh upon addition of water droplets and their stabilized water resistant structure after crosslinking with glutaraldehyde (Figures 9(c)-9(f)) [116]. Crosslinking in addition to stabilizing the membrane structure controls the thermochemical behaviour of the electrospun structure.

Several physical and chemical methods have been employed by researchers for effective crosslinking of the electrospun meshes, these include UV-irradiation, dehydrothermal treatment, and treatment with chemicals such as glutaraldehyde, formaldehyde, and carbodiimide [104, 105, $116,117]$. To avoid chemical toxicity of the retained chemical in the matrix, researchers prefer crosslinker treatment in vapour phase instead of the solution phase. Crosslinking the nanofiber during the electrospinning process, referred to as in situ crosslinking, is also reported in literature [118].

\section{Other Applications of Electrospun Nanofibers}

4.1. Biomedical. Electrospun nanofibers form a unique class of materials to be used for biomedical application. The submicron size and high surface area along with the porous architecture of nanofibers and the nanofiber mesh closely relates them to biostructures. Additionally, the flexibility of the technique to generate customized structures, with desirable surface and bulk properties, by utilizing blends and hybrids of different polymers and varying processing parameters and methods, has sparked interest in the electrospun polymers for biomedical application. In tissue engineering electrospun mats provide support for cell attachment and growth along with the exchange of nutrients and gases [119, 120]. An ideal three-dimensional scaffold should therefore have a highly porous structure with a large surface area $[59,121]$. Such a scaffold can be prepared by electrospinning $[6,61]$. The broad application of electrospun nanofibers in the field of biomedical, apart from the drug delivery include: tissue engineering [70, 122-124], wound dressing [125-127], enzyme immobilization [128-131], health care [92-95, 102], and biotechnology [55]. Extensive research in tissue engineering application targets the bone $[69,70$, $122,125]$, tendon and ligament $[67,68]$, vascular $[1,62,63$, 
123, 124], neural [64], and skin tissue [125-127]. Reports indicate two-thirds of electrospinning applications are in the biomedical field [23]. Composite scaffolds, in particular, are useful in tissue engineering for the reconstruction of structural tissues, multitissue organs, and tissue interfaces $[122,132,133]$. Hydroxyapatite/PLA composites have a high mechanical strength, good osteo-conductivity, osteoinductivity, and biodegradability $[69,134]$. In a study undertaken by $\mathrm{Xu}$ and coworkers [61], composite nanofibrous scaffolds composed of a poly(L-lactide)-grafted hydroxyapatite (PLA-g-HAP) nanoparticles and polylactide (PLA) matrix were prepared by electrospinning. The morphology of the composite nanofibers as well as the distribution of PLA-gHAP nanoparticles in the nanofibers was investigated using environmental scanning electron microscope (ESEM) and transmission electron microscopy (TEM). Results showed that at a low PLA-g-HAP content (approximately $4 \% \mathrm{~W} / \mathrm{V}$ ), the nanoparticles dispersed uniformly in the nanofibers and the composite nanofibrous mats exhibited higher strength properties, compared to the pristine PLA and the simple hydroxyapatite/PLA blended nanofiber mats. However, as the content of PLA-g-HAP increased, the nanoparticles began to aggregate, which resulted in the deterioration of the mechanical properties of the composite nanofiber mats. Erisken and coworkers [135], by employing the process of extrusion electrospinning, prepared nanocomposites of PCL and $\beta$-tricalcium phosphate ( $\beta$-TCP). Such composites with hydroxyapatite and $\beta$-TCP nanoparticles embedded in nanofibrous mats better mimicked the structural and functional abilities of bone tissue [61, 135]. In a different approach of Yang and coworkers [70] electrospun PCL scaffolds were surface treated with plasma. This technique efficiently introduced a bone-like mineralized apatite layer without compromising on the porous and fibrous integrity of the PCL scaffold [70].

Shalumon and coworkers [125] recently reported a scaffold of PCL and natural polymer chitosan (CS) by singlestep electrospinning. The authors reported the scaffold to support growth of various cell lines such as murine aneuploid fibro sarcoma (L929), human osteosarcoma cells (MG63), and mouse embryo fibroblasts (NIH3T3), exhibiting prospective application in skin and bone tissue engineering (Figure 10). Nanofibrous scaffolds of N-carboxyethyl chitosan (N-CECS)/PVA were prepared by electrospinning, and their potential as skin regeneration or wound dressing scaffold was evaluated in vitro. The nanofibrous mats supported good cell attachment and proliferation of mouse fibroblast cells $[126,127]$.

Lee and coworkers [123] prepared biocompatible vascular grafts by electrospinning PCL/collagen. The scaffold was reported to withstand high pressure flow up to a period of 4 weeks, depicting strong mechanical characteristics required for vascular constructs. The scaffold supported the growth of vascular cells. Another group of researchers prepared grafts of single polymer silk fibroin by electrospinning and coated it with silk sponge to enhance its mechanical strength. The authors report the cardiovascular application of this small diameter graft [124]. Zhou and coworkers [136] further reported successful preparation

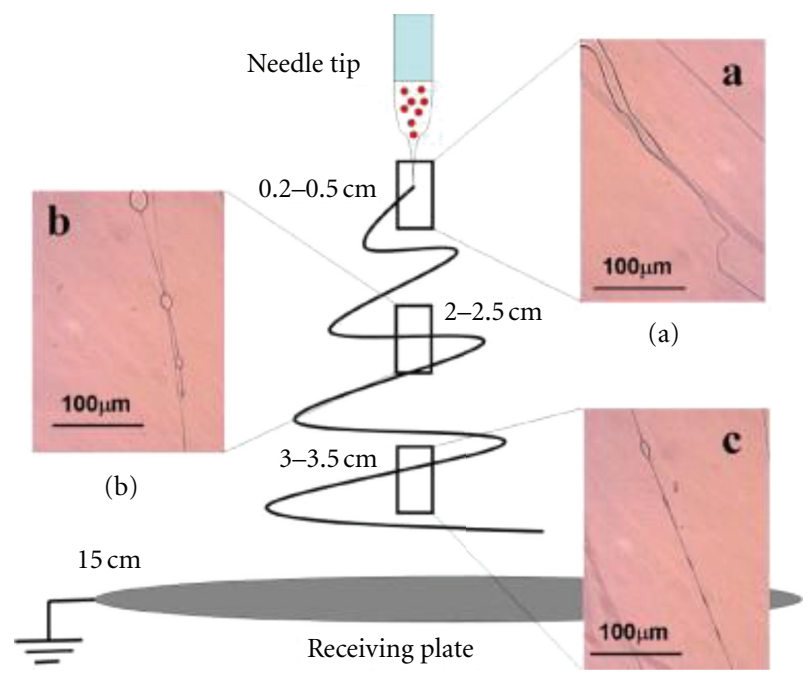

(c)

FIGURE 8: Morphological details of forming process during emulsion electrospinning. Optical images of the fibers $((a)-(c))$ collected on glass slide between the nozzle and collector as emulsion stretched into fibers at the distance of $1 \mathrm{~cm}, 2 \mathrm{~cm}$, and $4 \mathrm{~cm}$ from the electrospun Taylor cone. Adapted from Li et al. [114].

of tubular scaffold from electrospinning silk fibroin with collagen.

Enzyme immobilization on electrospun mats is also a well-researched field with an array of enzymes which have been successfully immobilized on nanofiber meshes of different polymer and subjected to different applications as in biosensor membrane, fermentation [128-131, 137, 138]. Reports suggest that incorporating biopolymer in the matrix provides a favorable environment for enzyme stability and retention of activity [137]. Electrospun nanofiber mats have potentially been used for immobilizing microbial cells. Pseudomonas nitroreducens LY immobilization on PVA mats has been achieved by electrospinning and applied for synthesizing theanine giving a yield of $10.75 \mathrm{~g} / \mathrm{L}$ [139].

4.2. Adsorption. Electrospun nanofiber mats are exceedingly useful in the field of adsorption and extraction. Oh and coworkers [48] produce nanofibers to remove toluene, which is a volatile organic compound that is considered to be a pollutant and therefore its level needs to be controlled in the atmosphere. The authors used electrospinning to produce nanofibers of polyacrylonitrile (PAN) which they then stabilized and carbonized to yield carbon nanofibers and activated them further at $1000^{\circ} \mathrm{C}$ to improve the toluene adsorption capacity. Activated carbon nanofibers (ACNF) exhibited excellent adsorption capacity of $65 \mathrm{~g}$-toluene $/ 100 \mathrm{~g}$-ACNF which they attributed to the high surface area $\left(1403 \mathrm{~m}^{2} / \mathrm{g}\right)$, large micropore volume $\left(0.505 \mathrm{~cm}^{3} / \mathrm{g}\right)$, and narrow average pore diameter $\left(6.0^{\circ} \mathrm{A}\right)$ of the ACNF. Kang and coworkers [140] developed a method of extracting trazodone from human plasma with an electrospun nanofiber solid-phase extraction sorbent. A polystyrene solution was electrospun and the nanofiber web was collected on a copper grid. 


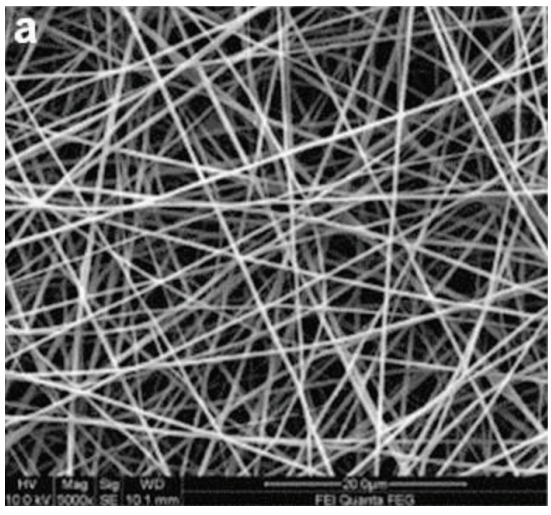

(a)

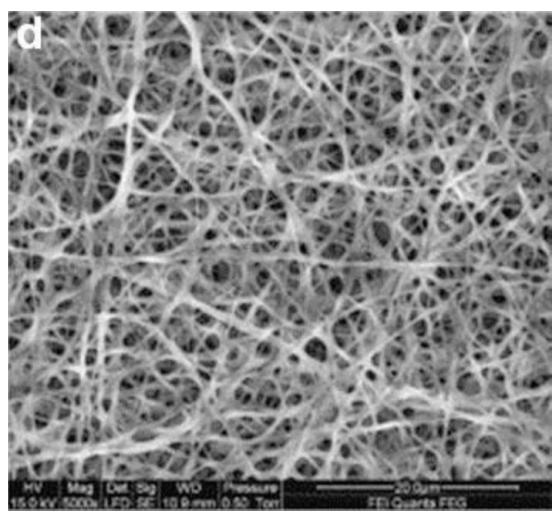

(d)

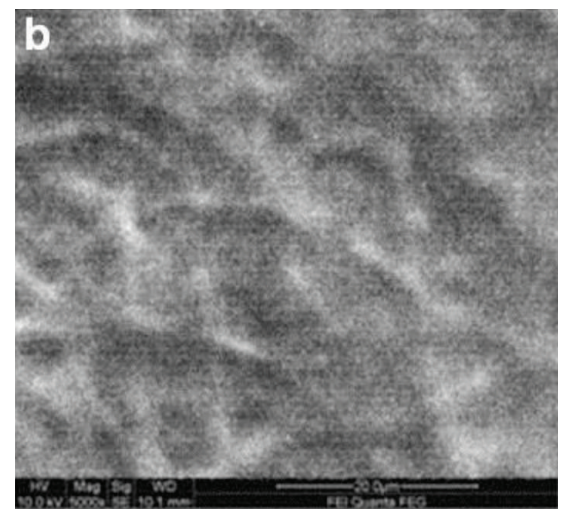

(b)

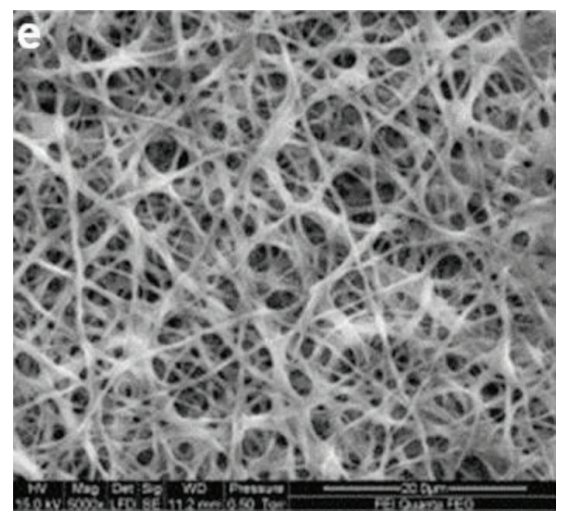

(e)

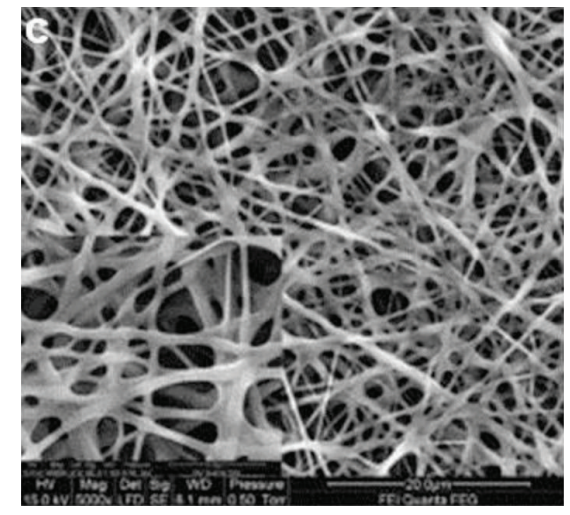

(c)

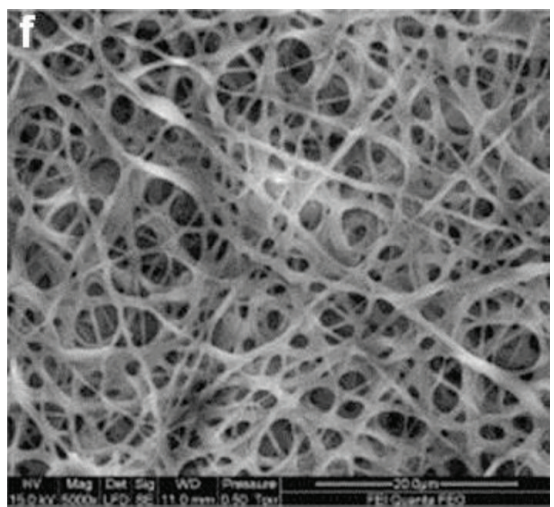

(f)

FIGURE 9: Morphologies of (a) as-spun gelatin nanofibers of a 10\% w/v gelatin/TFE, (b) the smeared surface layer of as-spun gelatin nanofibrous membrane after adding a drop of water, (c) cross-linked electrospun gelatin fibers before water-resistant test, ((d)-(f)) crosslinked electrospun gelatin fibers soaked in $37^{\circ} \mathrm{C}$ DI water for (d) 2 days (e), 4 days, and (f) 6 days (FESEM after drying for 1 week in a vacuum oven). Adapted from Zhang et al. [116].

Samples were pushed through this sorbent and trazodone was extracted. The sorbent was then washed with water and analytes were eluted with methanol. The methanol used for elution was analysed by high performance liquid chromatography (HPLC). This method of extraction and analysis of trazodone was found to be effective by the authors.

4.3. Sensors. Biosensors are the indispensable need of food industry, health care, environment sensing, and drug industry. This is an ever-advancing field with current focus on the development of compact and portable sensors without compromising on the sensitivity, which is a function of the analyte sensing/adsorbing membrane. Electrospinning resolves both issues by fabricating ultrafine nanofibers in submicron range and with high porosity. A many-fold high surface to volume ratio is achievable in electrospun nanofibers compared to any other counterpart of similar volume. Such surfaces are efficient in sensing minute quantities of analytes, such as gases, as high surface area allows for higher adsorption/absorption, thereby improving on the sensitivity while being compact in dimensions. Electrospun membranes have been used in biosensors for the detection of urea, glucose, cauliflower mosaic virus, DNA, cholesterol, hydrogen peroxides, catechol, amines, alcohols, nitrogen dioxide, and carbon monoxide [51, 141-149]. A range of polymers that has proved useful in fabricating biosensing nanofiber mats include polyaniline, polyamic acid, polypyrrole, nylon-6, PVA, and chitosan [145, 146, 150-154]. Researchers are further exploring nanofiber mats application in electrochemical and optical sensors but much progress has not been reported so far $[23,151]$.

4.4. Filtration. The unmatched property of electrospinning technique to produce ultrathin nanofibers has been tapped way back, for producing air and liquid filtration membranes. The nonwoven electrospun mats are being efficiently used as high-performance air filters. The high surface to volume ratio and cohesive properties of polymers nanofibers play a pivotal role in removing airborne particles of size as small as 1-5 $\mu \mathrm{m}[155,156]$. In a specified approach towards increasing the filtration efficiency of nanofiber mats without elevating the pressure drop, charge was introduced on fiber surface [46]. 

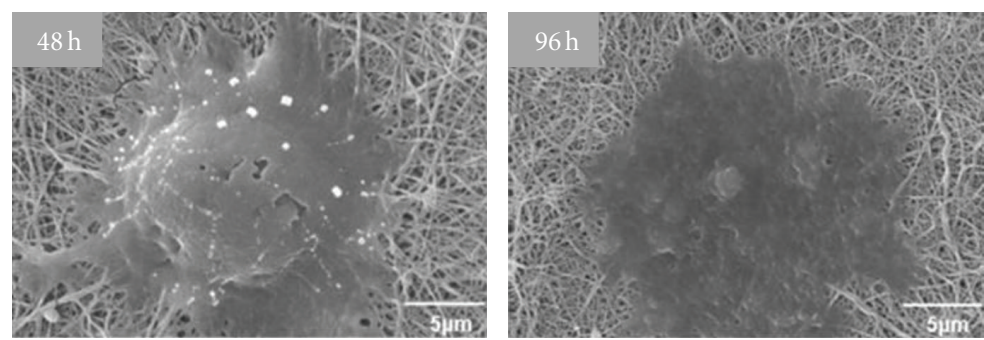

(a)
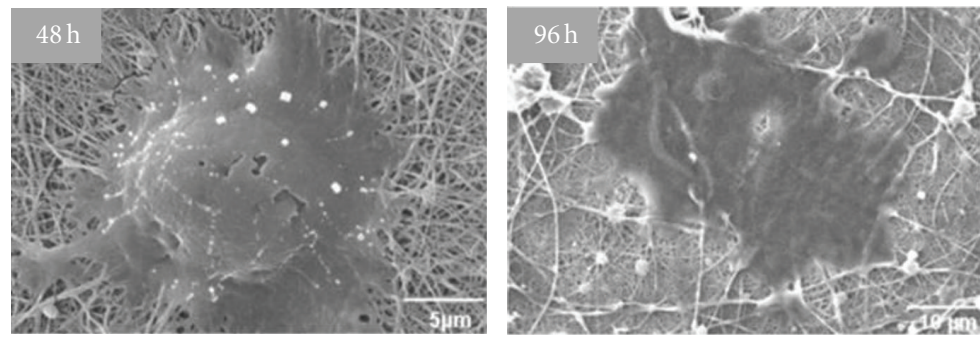

(b)
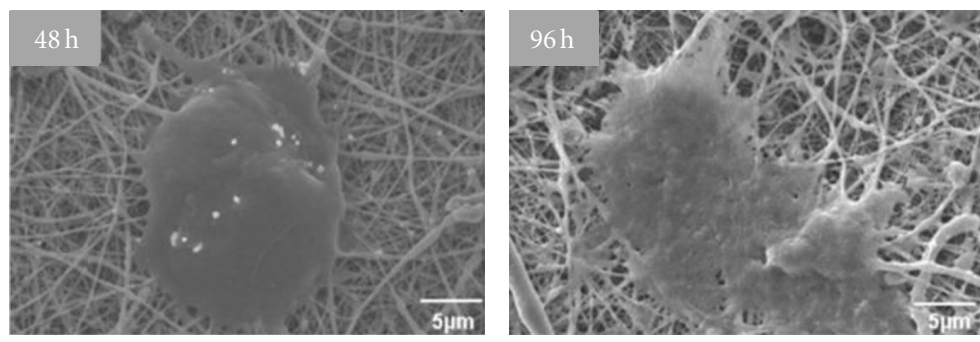

(c)

FIGURE 10: SEM images of electrospun CS/PCL nanofibrous scaffold supporting cell attachment using (a) human osteoscarcoma cells (MG63), (b) murine aneuploid fibro sarcoma (L929), and (c) mouse embryo fibroblasts (NIH3T3). Adapted from Shalumon et al. [125].

Ahn and coworkers [45] developed nanofilters by electrospinning Nylon 6. Authors evaluated the filtration efficiency and pressure drop of the nanofiber filters and compared it to that of HEPA filters. They reported a $99.993 \%$ superior filtration efficiency of the developed nylon 6 nanofilters, thereby proving the potential of developed filters as high efficiency filters. Electrospun mats have additionally found application as antimicrobial filters. Electrospun cationomers with quaternary ammonium groups have successfully been used as nanofilters against pathogenic bacterial strains of Staphylococcus aureus and Escherichia coli $[157,158]$.

\section{Conclusions}

The nanotechnology boom has catalyzed the exploitation of techniques that can be employed for preparing nanomaterials with various applications. Although the technique of electrospinning was discovered years ago, but its rediscovery, through the work of Formhals in 1934, has gathered much interest as a simple and versatile technical tool for the preparation of nanofibers. As described in this paper the technique offers the possibility of tuning nanofiber morphology, porosity and topography in particular dimensions, structural form, and density. These characteristics of nanofibers and nanofiber meshes can be controlled and altered in accordance with their end-point application by varying, evaluating, and optimizing different formulation parameters of the electrospinning process. By modifying such parameters the porosity of the nanofibers can be controlled as well as the nanofiber diameter, density, and overall morphology. The paper has concisely demonstrated that for the majority of electrospinning formulation variables there is a permissible window/range within which electrospinning of nanofibers is favoured and out of these extremities defects are observed. The bulk and surface properties of electrospun nanofibers are, in the first place, governed by the choice of the electrospinning material. A diverse class of polymers have so far been reported to be electrospun as a solution or melt. The limitless possibilities of electrospinning polymers include the fabrication of nanofiber from polymer blends, hybrids, or even composites. For the electrospun polymeric mesh to closely mimic a specified biological structure and its features or to display structural and functional properties as required for the end use of it, a single polymer or combination of polymers can be 
electrospun. Such versatility of the properties of electrospun nanofiber structures opens up plethora of diverse fields for their application, such as in drug delivery, tissue engineering, wound dressing, filtration, enzyme immobilization, and even as sensors. Although new drug delivery systems based on electrospinning are being formulated, there is still scope for much extensive research on different electrospinning parameters and to develop techniques for electrospinning new polymer combinations. Novel methods for loading a variety of drugs and other bioactivities in electrospun nanofibers need to be exploited further to fulfil the growing demand of the need for drug delivery systems.

\section{Conflict of Interests}

The authors have no conflict of interests.

\section{References}

[1] Z. Ma, M. Kotaki, T. Yong, W. He, and S. Ramakrishna, "Surface engineering of electrospun polyethylene terephthalate (PET) nanofibers towards development of a new material for blood vessel engineering," Biomaterials, vol. 26, no. 15, pp. 2527-2536, 2005.

[2] J. P. Chen, G. Y. Chang, and J. K. Chen, "Electrospun collagen/chitosan nanofibrous membrane as wound dressing," Colloids and Surfaces A, vol. 313-314, pp. 183-188, 2008.

[3] T. G. Kim, D. S. Lee, and T. G. Park, "Controlled protein release from electrospun biodegradable fiber mesh composed of poly( $\varepsilon$-caprolactone) and poly(ethylene oxide)," International Journal of Pharmaceutics, vol. 338, no. 1-2, pp. 276-283, 2007.

[4] S. Maretschek, A. Greiner, and T. Kissel, "Electrospun biodegradable nanofiber nonwovens for controlled release of proteins," Journal of Controlled Release, vol. 127, no. 2, pp. 180-187, 2008.

[5] G. Verreck, I. Chun, J. Rosenblatt et al., "Incorporation of drugs in an amorphous state into electrospun nanofibers composed of a water-insoluble, nonbiodegradable polymer," Journal of Controlled Release, vol. 92, no. 3, pp. 349-360, 2003.

[6] X. L. Deng, G. Sui, M. L. Zhao, G. Q. Chen, and X. P. Yang, "Poly(L-lactic acid)/hydroxyapatite hybrid nanofibrous scaffolds prepared by electrospinning," Journal of Biomaterials Science, vol. 18, no. 1, pp. 117-130, 2007.

[7] P. X. Ma and R. Zhang, "Synthetic nano-scale fibrous extracellular matrix," Journal of Biomedical Materials Research, vol. 46, no. 1, pp. 60-72, 1999.

[8] D. Liu, H. Zhang, P. C. M. Grim et al., "Self-assembly of polyphenylene dendrimers into micrometer long nanofibers: an atomic force microscopy study," Langmuir, vol. 18, no. 6, pp. 2385-2391, 2002.

[9] G. Liu, L. Qiao, and A. Guo, "Diblock copolymer nanofibers," Macromolecules, vol. 29, no. 16, pp. 5508-5510, 1996.

[10] X. Yan, G. Liu, F. Liu et al., "Superparamagnetic triblock copolymer $/ \mathrm{Fe}_{2} \mathrm{O}_{3}$ hybrid nanofibers," Angewandte Chemie International Edition, vol. 40, no. 19, pp. 3593-3596, 2001.

[11] C.-G. Wu and T. Bein, "Conducting polyaniline filaments in a mesoporous channel host," Science, vol. 264, no. 5166, pp. 17571759, 1994.

[12] L. Feng, S. Li, H. Li et al., "Super-hydrophobic surface of aligned polyacrylonitrile nanofibers," Angewandte Chemie International Edition, vol. 41, no. 7, pp. 1221-1223, 2002.
[13] T. Ondarcuhu and C. Joachim, "Drawing a single nanofiber over hundreds of microns," Europhysics Letters, vol. 42, no. 2, pp. 215220, 1998.

[14] P. K. Baumgarten, "Electrostatic spinning of acrylic microfibers," Journal of Colloid and Interface Science, vol. 36, no. 1, pp. 71-79, 1971.

[15] J. Doshi and D. H. Reneker, "Electrospinning process and applications of electrospun fibers," Journal of Electrostatics, vol. 35, no. 2-3, pp. 151-160, 1995.

[16] G. Taylor, "Electrically driven jets," Proceedings of the Royal Society of London A, vol. 313, no. 1515, pp. 453-475, 1969.

[17] D. Liang, B. S. Hsiao, and B. Chu, "Functional electrospun nanofibrous scaffolds for biomedical applications," Advanced Drug Delivery Reviews, vol. 59, no. 14, pp. 1392-1412, 2007.

[18] G. G. Wallace, M. J. Higgins, S. E. Moulton, and C. Wang, "Nanobionics: the impact of nanotechnology on implantable medical bionic devices," Nanoscale, vol. 4, no. 15, pp. 4327-4347, 2012.

[19] D. H. Reneker and I. Chun, "Nanometre diameter fibres of polymer, produced by electro-spinning," Nanotechnology, vol. 7, no. 3, pp. 216-223, 1996.

[20] D. Li and Y. Xia, "Electrospinning of nanofibers: reinventing the wheel?" Advanced Materials, vol. 16, no. 14, pp. 1151-1170, 2004.

[21] M. M. Hohman, M. Shin, G. Rutledge, and M. P. Brenner, "Electrospinning and electrically forced jets. I. Stability theory," Physics of Fluids, vol. 13, no. 8, pp. 2201-2220, 2001.

[22] J. M. Deitzel, J. Kleinmeyer, D. Harris, and N. C. Beck Tan, “The effect of processing variables on the morphology of electrospun nanofibers and textiles," Polymer, vol. 42, no. 1, pp. 261-272, 2001.

[23] N. Bhardwaj and S. C. Kundu, "Electrospinning: a fascinating fiber fabrication technique," Biotechnology Advances, vol. 28, no. 3, pp. 325-347, 2010.

[24] J. Zeleny, “The electrical discharge from liquid points, and a hydrostatic method of measuring the electric intensity at their surfaces," Physical Review, vol. 3, no. 2, pp. 69-91, 1914.

[25] J. Zeleny, "Instability of electrified liquid surfaces," Physical Review, vol. 10, no. 1, pp. 1-6, 1917.

[26] J. Zeleny, "The role of surface instability in electrical discharges from drops of alcohol and water in air at atmospheric pressure," Journal of the Franklin Institute, vol. 219, no. 6, pp. 659-675, 1935.

[27] A. Formhals, "Process and apparatus for preparing artificial threads," US Patent 1-975-504, 1934.

[28] A. Formhals, "Method and apparatus for spinning," US Patent 2-160-962, 1939.

[29] A. Formhals, "Artificial thread and method of producing same," US Patent 2-187-306, 1940.

[30] A. Formhals, "Production of artificial fibres from fibre forming liquids," US Patent 2-323-025, 1934.

[31] L. Larrondo and R. S. J. Manley, "Electrostatic fiber spinning from polymer melts. 1. Experimental-observations on fiber formation and properties," Journal of Polymer Science A, vol. 19, no. 6, pp. 909-920, 1981.

[32] L. Larrondo and R. S. J. Manley, "Electrostatic fiber spinning from polymer melts. 2. Examination of the flow field in an electrically driven jet," Journal of Polymer Science A, vol. 19, no. 6, pp. 921-932, 1981.

[33] W. Yao, J. Yang, J. Wang, and L. Tao, "Synthesis and electrochemical performance of carbon nanofiber-cobalt oxide composites," Electrochimica Acta, vol. 53, no. 24, pp. 7326-7330, 2008. 
[34] W. L. Yao, J. L. Wang, J. Yang, and G. D. Du, "Novel carbon nanofiber-cobalt oxide composites for lithium storage with large capacity and high reversibility," Journal of Power Sources, vol. 176, no. 1, pp. 369-372, 2008.

[35] A. G. MacDiarmid, W. E. Jones, I. D. Norris et al., "Electrostatically-generated nanofibers of electronic polymers," Synthetic Metals, vol. 119, no. 1-3, pp. 27-30, 2001.

[36] P. Viswanathamurthi, N. Bhattarai, H. Y. Kim, and D. R. Lee, "Vanadium pentoxide nanofibers by electrospinning," Scripta Materialia, vol. 49, no. 6, pp. 577-581, 2003.

[37] A. Allaoui, S. V. Hoa, and M. D. Pugh, "The electronic transport properties and microstructure of carbon nanofiber/epoxy composites," Composites Science and Technology, vol. 68, no. 2, pp. 410-416, 2008.

[38] Y. Xin, Z. Huang, W. Li, Z. Jiang, Y. Tong, and C. Wang, "Coresheath functional polymer nanofibers prepared by coelectrospinning," European Polymer Journal, vol. 44, no. 4, pp. 1040-1045, 2008.

[39] Y. W. Ju, J. H. Park, H. R. Jung, S. J. Cho, and W. J. Lee, "Electrospun $\mathrm{MnFe}_{2} \mathrm{O}_{4}$ nanofibers: preparation and morphology," Composites Science and Technology, vol. 68, no. 7-8, pp. 17041709, 2008.

[40] S. Tan, X. Feng, B. Zhao, Y. Zou, and X. Huang, "Preparation and photoluminescence properties of electrospun nanofibers containing $\mathrm{PMO}-\mathrm{PPV}$ and $\mathrm{Eu}(\mathrm{ODBM})_{3}$ phen," Materials Letters, vol. 62, no. 16, pp. 2419-2421, 2008.

[41] J. Gong, C. L. Shao, G. C. Yang, Y. Pan, and L. Y. Qu, "Preparation of ultra-fine fiber mats contained $\mathrm{H}_{4} \mathrm{SiW}_{12} \mathrm{O}_{40}$ ", Inorganic Chemistry Communications, vol. 6, no. 7, pp. 916-918, 2003.

[42] J. Bai, Y. Li, M. Li, S. Wang, C. Zhang, and Q. Yang, "Electrospinning method for the preparation of silver chloride nanoparticles in PVP nanofiber," Applied Surface Science, vol. 254, no. 15, pp. 4520-4523, 2008.

[43] M. Li, G. Han, and B. Yang, "Fabrication of the catalytic electrodes for methanol oxidation on electrospinning-derived carbon fibrous mats," Electrochemistry Communications, vol. 10, no. 6, pp. 880-883, 2008.

[44] S. H. Park, C. Kim, Y. O. Choi, and K. S. Yang, "Preparations of pitch-based CF/ACF webs by electrospinning," Carbon, vol. 41, no. 13, pp. 2655-2657, 2003.

[45] Y. C. Ahn, S. K. Park, G. T. Kim et al., "Development of high efficiency nanofilters made of nanofibers," Current Applied Physics, vol. 6, no. 6, pp. 1030-1035, 2006.

[46] P. P. Tsai, H. Schreuder-Gibson, and P. Gibson, "Different electrostatic methods for making electret filters," Journal of Electrostatics, vol. 54, no. 3-4, pp. 333-341, 2002.

[47] A. A. Ali, "New generation of super absorber nano-fibroses hybrid fabric by electro-spinning," Journal of Materials Processing Technology, vol. 199, no. 1, pp. 193-198, 2008.

[48] G. Y. Oh, Y. W. Ju, M. Y. Kim et al., "Adsorption of toluene on carbon nanofibers prepared by electro-spinning," Science of the Total Environment, vol. 393, no. 2-3, pp. 341-347, 2008.

[49] B. Ding, J. Kim, Y. Miyazaki, and S. Shiratori, "Electrospun nanofibrous membranes coated quartz crystal microbalance as gas sensor for $\mathrm{NH}_{3}$ detection," Sensors and Actuators B, vol. 101, no. 3, pp. 373-380, 2004.

[50] Z. F. Li, F. D. Blum, M. F. Bertino, C. S. Kim, and S. K. Pillalamarri, "One-step fabrication of a polyaniline nanofiber vapor sensor," Sensors and Actuators B, vol. 134, no. 1, pp. 31-35, 2008.
[51] K. Sawicka, P. Gouma, and S. Simon, "Electrospun biocomposite nanofibers for urea biosensing," Sensors and Actuators B, vol. 108, no. 1-2, pp. 585-588, 2005.

[52] M. Song, C. Pan, J. Li, R. Zhang, X. Wang, and Z. Gu, "Blends of $\mathrm{TiO}_{2}$ nanoparticles and poly (N-isopropylacrylamide)-copolystyrene nanofibers as a means to promote the biorecognition of an anticancer drug," Talanta, vol. 75, no. 4, pp. 1035-1040, 2008.

[53] E. R. Kenawy, F. I. Abdel-Hay, M. H. El-Newehy, and G. E. Wnek, "Controlled release of ketoprofen from electrospun poly(vinyl alcohol) nanofibers," Materials Science and Engineering A, vol. 459, no. 1-2, pp. 390-396, 2007.

[54] S. Tungprapa, I. Jangchud, and P. Supaphol, "Release characteristics of four model drugs from drug-loaded electrospun cellulose acetate fiber mats," Polymer, vol. 48, no. 17, pp. 50305041, 2007.

[55] D. Liang, Y. K. Luu, K. Kim, B. S. Hsiao, M. Hadjiargyrou, and B. Chu, "In vitro non-viral gene delivery with nanofibrous scaffolds," Nucleic Acids Research, vol. 33, no. 19, article e170, 2005.

[56] B. Chu, D. Liang, M. Hadjiargyrou, and B. S. Hsiao, "A new pathway for developing in vitro nanostructured non-viral gene carriers," Journal of Physics Condensed Matter, vol. 18, no. 36, supplement, pp. S2513-S2525, 2006.

[57] X. Zong, S. Li, E. Chen et al., "Prevention of postsurgeryinduced abdominal adhesions by electrospun bioabsorbable nanofibrous poly(lactide-co-glycolide)-based membranes," Annals of Surgery, vol. 240, no. 5, pp. 910-915, 2004.

[58] M. S. Khil, D. I. Cha, H. Y. Kim, I. S. Kim, and N. Bhattarai, "Electrospun nanofibrous polyurethane membrane as wound dressing," Journal of Biomedical Materials Research B, vol. 67, no. 2, pp. 675-679, 2003.

[59] Q. Z. Chen, I. D. Thompson, and A. R. Boccaccini, “45S5 Bioglass-derived glass-ceramic scaffolds for bone tissue engineering," Biomaterials, vol. 27, no. 11, pp. 2414-2425, 2006.

[60] H. Yoshimoto, Y. M. Shin, H. Terai, and J. P. Vacanti, "A biodegradable nanofiber scaffold by electrospinning and its potential for bone tissue engineering," Biomaterials, vol. 24, no. 12, pp. 2077-2082, 2003.

[61] X. Xu, X. Chen, A. Liu, Z. Hong, and X. Jing, "Electrospun poly(l-lactide)-grafted hydroxyapatite/poly(l-lactide) nanocomposite fibers," European Polymer Journal, vol. 43, no. 8, pp. 3187-3196, 2007.

[62] C. Y. Xu, R. Inai, M. Kotaki, and S. Ramakrishna, "Aligned biodegradable nanofibrous structure: a potential scaffold for blood vessel engineering," Biomaterials, vol. 25, no. 5, pp. 877886, 2004.

[63] C. M. Vaz, S. van Tuijl, C. V. C. Bouten, and F. P. T. Baaijens, "Design of scaffolds for blood vessel tissue engineering using a multi-layering electrospinning technique," Acta Biomaterialia, vol. 1, no. 5, pp. 575-582, 2005.

[64] F. Yang, R. Murugan, S. Wang, and S. Ramakrishna, "Electrospinning of nano/micro scale poly(l-lactic acid) aligned fibers and their potential in neural tissue engineering," Biomaterials, vol. 26, no. 15, pp. 2603-2610, 2005.

[65] E. Schnell, K. Klinkhammer, S. Balzer et al., "Guidance of glial cell migration and axonal growth on electrospun nanofibers of

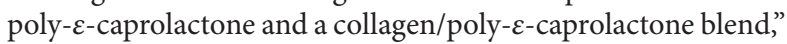
Biomaterials, vol. 28, no. 19, pp. 3012-3025, 2007.

[66] S. Y. Chew, R. Mi, A. Hoke, and K. W. Leong, "The effect of the alignment of electrospun fibrous scaffolds on Schwann cell maturation," Biomaterials, vol. 29, no. 6, pp. 653-661, 2008. 
[67] C. H. Lee, H. J. Shin, I. H. Cho et al., "Nanofiber alignment and direction of mechanical strain affect the ECM production of human ACL fibroblast," Biomaterials, vol. 26, no. 11, pp. 12611270, 2005.

[68] S. Sahoo, H. Ouyang, C. H. James, T. E. Tay, and S. L. Toh, "Characterization of a novel polymeric scaffold for potential application in tendon/ligament tissue engineering," Tissue Engineering, vol. 12, no. 1, pp. 91-99, 2006.

[69] S. Liao, R. Murugan, C. K. Chan, and S. Ramakrishna, "Processing nanoengineered scaffolds through electrospinning and mineralization suitable for biomimetic bone tissue engineering," Journal of the Mechanical Behavior of Biomedical Materials, vol. 1, no. 3, pp. 252-260, 2008.

[70] F. Yang, J. G. C. Wolke, and J. A. Jansen, "Biomimetic calcium phosphate coating on electrospun poly( $\varepsilon$-caprolactone) scaffolds for bone tissue engineering," Chemical Engineering Journal, vol. 137, no. 1, pp. 154-161, 2008.

[71] J. C. J. Hogan and P. Biswas, "Narrow size distribution nanoparticle production by electrospray processing of ferritin," Journal of Aerosol Science, vol. 39, no. 5, pp. 432-440, 2008.

[72] A. Jaworek, "Micro- and nanoparticle production by electrospraying," Powder Technology, vol. 176, no. 1, pp. 18-35, 2007.

[73] T. Subbiah, G. S. Bhat, R. W. Tock, S. Parameswaran, and S. S. Ramkumar, "Electrospinning of nanofibers," Journal of Applied Polymer Science, vol. 96, no. 2, pp. 557-569, 2005.

[74] T. J. Sill and H. A. von Recum, "Electrospinning: applications in drug delivery and tissue engineering," Biomaterials, vol. 29, no. 13, pp. 1989-2006, 2008.

[75] A. K. Haghi and M. Akbari, "Trends in electrospinning of natural nanofibers," Physica Status Solidi (A) Applications and Materials, vol. 204, no. 6, pp. 1830-1834, 2007.

[76] K. J. Pawlowski, C. P. Barnes, E. D. Boland, G. E. Wnek, and G. L. Bowlin, "Biomedical nanoscience: electrospinning basic concepts, applications, and classroom demonstration," Materials Research Society Symposium Proceedings, vol. 827, pp. 17-28, 2004.

[77] C. Meechaisue, R. Dubin, P. Supaphol, V. P. Hoven, and J. Kohn, "Electrospun mat of tyrosine-derived polycarbonate fibers for potential use as tissue scaffolding material," Journal of Biomaterials Science, vol. 17, no. 9, pp. 1039-1056, 2006.

[78] X. Zong, K. Kim, D. Fang, S. Ran, B. S. Hsiao, and B. Chu, "Structure and process relationship of electrospun bioabsorbable nanofiber membranes," Polymer, vol. 43, no. 16, pp. 4403-4412, 2002.

[79] S. Megelski, J. S. Stephens, D. Bruce Chase, and J. F. Rabolt, "Micro- and nanostructured surface morphology on electrospun polymer fibers," Macromolecules, vol. 35, no. 22, pp. 84568466, 2002.

[80] S. A. Theron, E. Zussman, and A. L. Yarin, "Experimental investigation of the governing parameters in the electrospinning of polymer solutions," Polymer, vol. 45, no. 6, pp. 2017-2030, 2004.

[81] J. Venugopal, Y. Z. Zhang, and S. Ramakrishna, "Electrospun nanofibers: biomedical applications," Proceedings of the Institution of Mechanical Engineers N, vol. 218, pp. 35-45, 2005.

[82] A. Greiner and J. H. Wendorff, "Electrospinning: a fascinating method for the preparation of ultrathin fibers," Angewandte Chemie, vol. 46, no. 30, pp. 5670-5703, 2007.

[83] H. Fong, I. Chun, and D. H. Reneker, "Beaded nanofibers formed during electrospinning," Polymer, vol. 40, no. 16, pp. 4585-4592, 1999.
[84] J. Lannutti, D. Reneker, T. Ma, D. Tomasko, and D. Farson, "Electrospinning for tissue engineering scaffolds," Materials Science and Engineering C, vol. 27, no. 3, pp. 504-509, 2007.

[85] Q. Yang, L. I. Zhenyu, Y. Hong et al., "Influence of solvents on the formation of ultrathin uniform poly(vinyl pyrrolidone) nanofibers with electrospinning," Journal of Polymer Science B, vol. 42, no. 20, pp. 3721-3726, 2004.

[86] W. K. Son, J. H. Youk, T. S. Lee, and W. H. Park, “The effects of solution properties and polyelectrolyte on electrospinning of ultrafine poly(ethylene oxide) fibers," Polymer, vol. 45, no. 9, pp. 2959-2966, 2004.

[87] T. Jarusuwannapoom, W. Hongrojjanawiwat, S. Jitjaicham et al., "Effect of solvents on electro-spinnability of polystyrene solutions and morphological appearance of resulting electrospun polystyrene fibers," European Polymer Journal, vol. 41, no. 3, pp. 409-421, 2005.

[88] L. Huang, K. Nagapudi, P. R. Apkarian, and E. L. Chaikof, "Engineered collagen-PEO nanofibers and fabrics," Journal of Biomaterials Science, vol. 12, no. 9, pp. 979-993, 2001.

[89] Y. H. Jung, H. Y. Kim, D. R. Lee, and S. Y. Park, "Characterization of $\mathrm{PVOH}$ nonwoven mats prepared from surfactantpolymer system via electrospinning," Macromolecular Research, vol. 13, no. 5, pp. 385-390, 2005.

[90] T. Lin, H. Wang, H. Wang, and X. Wang, “The charge effect of cationic surfactants on the elimination of fibre beads in the electrospinning of polystyrene," Nanotechnology, vol. 15, no. 9, pp. 1375-1381, 2004.

[91] J. Zeng, X. Xu, X. Chen et al., "Biodegradable electrospun fibers for drug delivery," Journal of Controlled Release, vol. 92, no. 3, pp. 227-231, 2003.

[92] Z. M. Huang, C. L. He, A. Yang et al., "Encapsulating drugs in biodegradable ultrafine fibres through co-axial electrospinning," Journal of Biomedical Materials Research A, vol. 77, no. 1, pp. 169-179, 2006.

[93] K. Kim, Y. K. Luu, C. Chang et al., "Incorporation and controlled release of a hydrophilic antibiotic using poly(lactideco-glycolide)-based electrospun nanofibrous scaffolds," Journal of Controlled Release, vol. 98, no. 1, pp. 47-56, 2004.

[94] X. Xu, X. Chen, P. Ma, X. Wang, and X. Jing, "The release behavior of doxorubicin hydrochloride from medicated fibers prepared by emulsion-electrospinning," European Journal of Pharmaceutics and Biopharmaceutics, vol. 70, no. 1, pp. 165-170, 2008.

[95] S. H. Ranganath and C. H. Wang, "Biodegradable microfiber implants delivering paclitaxel for post-surgical chemotherapy against malignant glioma," Biomaterials, vol. 29, no. 20, pp. 2996-3003, 2008.

[96] S. Y. Chew, J. Wen, E. K. F. Yim, and K. W. Leong, "Sustained release of proteins from electrospun biodegradable fibers," Biomacromolecules, vol. 6, no. 4, pp. 2017-2024, 2005.

[97] C. Burger and B. Chu, "Functional nanofibrous scaffolds for bone reconstruction," Colloids and Surfaces B, vol. 56, no. 1-2, pp. 134-141, 2007.

[98] M. Prabaharan, R. Jayakumar, and S. V. Nair, "Electrospun nanofibrous scaffolds-current status and prospects in drug delivery," Advances in Polymer Science, vol. 246, pp. 241-262, 2012.

[99] E. Luong-Van, L. Grøndahl, K. N. Chua, K. W. Leong, V. Nurcombe, and S. M. Cool, "Controlled release of heparin from poly( $\varepsilon$-caprolactone) electrospun fibers," Biomaterials, vol. 27, no. 9, pp. 2042-2050, 2006. 
[100] C. L. He, Z. M. Huang, X. J. Han, L. Liu, H. S. Zhang, and L. S. Chen, "Coaxial electrospun poly(L-lactic acid) ultrafine fibers for sustained drug delivery," Journal of Macromolecular Science $B$, vol. 45, no. 4, pp. 515-524, 2006.

[101] P. Taepaiboon, U. Rungsardthong, and P. Supaphol, "Drugloaded electrospun mats of poly(vinyl alcohol) fibres and their release characteristics of four model drugs," Nanotechnology, vol. 17, no. 9, pp. 2317-2329, 2006.

[102] P. Taepaiboon, U. Rungsardthong, and P. Supaphol, "Vitaminloaded electrospun cellulose acetate nanofiber mats as transdermal and dermal therapeutic agents of vitamin A acid and vitamin E," European Journal of Pharmaceutics and Biopharmaceutics, vol. 67, no. 2, pp. 387-397, 2007.

[103] O. Suwantong, P. Opanasopit, U. Ruktanonchai, and P. Supaphol, "Electrospun cellulose acetate fiber mats containing curcumin and release characteristic of the herbal substance," Polymer, vol. 48, no. 26, pp. 7546-7557, 2007.

[104] J. Zeng, L. Yang, Q. Liang et al., "Influence of the drug compatibility with polymer solution on the release kinetics of electrospun fiber formulation," Journal of Controlled Release, vol. 105, no. 1-2, pp. 43-51, 2005.

[105] D. Yang, Y. Li, and J. Nie, "Preparation of gelatin/PVA nanofibers and their potential application in controlled release of drugs," Carbohydrate Polymers, vol. 69, no. 3, pp. 538-543, 2007.

[106] H. Qi, P. Hu, J. Xu, and A. Wang, "Encapsulation of drug reservoirs in fibers by emulsion electrospinning: morphology characterization and preliminary release assessment," Biomacromolecules, vol. 7, no. 8, pp. 2327-2330, 2006.

[107] E. R. Kenawy, G. L. Bowlin, K. Mansfield et al., "Release of tetracycline hydrochloride from electrospun poly(ethyleneco-vinylacetate), poly(lactic acid), and a blend," Journal of Controlled Release, vol. 81, no. 1-2, pp. 57-64, 2002.

[108] X. Xu, L. Yang, X. Xu et al., "Ultrafine medicated fibers electrospun from W/O emulsions," Journal of Controlled Release, vol. 108, no. 1, pp. 33-42, 2005.

[109] A. Chunder, S. Sarkar, Y. Yu, and L. Zhai, "Fabrication of ultrathin polyelectrolyte fibers and their controlled release properties," Colloids and Surfaces B, vol. 58, no. 2, pp. 172-179, 2007.

[110] C. He, Z. Huang, and X. Han, "Fabrication of drug-loaded electrospun aligned fibrous threads for suture applications," Journal of Biomedical Materials Research B, vol. 89, no. 1, pp. 8095, 2008.

[111] X. Li, H. Zhang, H. Li, G. Tang, Y. Zhao, and X. Yuan, "Self-accelerated biodegradation of electrospun poly(ethylene glycol)-poly(l-lactide) membranes by loading proteinase K," Polymer Degradation and Stability, vol. 93, no. 3, pp. 618-626, 2008.

[112] M. Jacoby, "Hollow nanofibers in a single step: electrospinning, sol-gel chemistry are combined to form nanotubular fibers," Chemical and Engineering New, vol. 82, no. 17, p. 6, 2004.

[113] C. Wang, K. W. Yan, Y. D. Lin, and P. C. H. Hsieh, "Biodegradable core/shell fibers by coaxial electrospinning: processing, fiber characterization, and its application in sustained drug release," Macromolecules, vol. 43, no. 15, pp. 6389-6397, 2010.

[114] X. Li, Y. Su, S. Liu, L. Tan, X. Mo, and S. Ramakrishna, "Encapsulation of proteins in poly(l-lactide-co-caprolactone) fibers by emulsion electrospinning," Colloids and Surfaces B, vol. 75, no. 2, pp. 418-424, 2010.
[115] N. Bölgen, I. Vargel, P. Korkusuz, Y. Z. Menceloğlu, and E. Pișkin, "In vivo performance of antibiotic embedded electrospun PCL membranes for prevention of abdominal adhesions," Journal of Biomedical Materials Research B, vol. 81, no. 2, pp. 530-543, 2007.

[116] Y. Z. Zhang, J. Venugopal, Z. M. Huang, C. T. Lim, and S. Ramakrishna, "Crosslinking of the electrospun gelatin nanofibers," Polymer, vol. 47, no. 8, pp. 2911-2917, 2006.

[117] R. P. Shaikh, P. Kumar, Y. E. Choonara, L. C. du Toit, and V. Pillay, "Crosslinked electrospun PVA nanofibrous membranes: elucidation of their physicochemical, physicomechanical and molecular disposition," Biofabrication, vol. 4, Article ID 025002, 2 pages, 2012.

[118] C. Tang, C. D. Saquing, J. R. Harding, and S. A. Khan, "In situ cross-linking of electrospun poly(vinyl alcohol) nanofibers," Macromolecules, vol. 43, no. 2, pp. 630-637, 2010.

[119] B. Sharma and J. H. Elisseeff, "Engineering structurally organized cartilage and bone tissues," Annals of Biomedical Engineering, vol. 32, no. 1, pp. 148-159, 2004.

[120] X. Liu and P. X. Ma, "Polymeric scaffolds for bone tissue engineering," Annals of Biomedical Engineering, vol. 32, no. 3, pp. 477-486, 2004.

[121] B. Viswanath and N. Ravishankar, "Porous biphasic scaffolds and coatings for biomedical applications via morphology transition of nanorods," Nanotechnology, vol. 18, no. 47, Article ID 475604, 2007.

[122] V. Maquet, A. R. Boccaccini, L. Pravata, I. Notingher, and R. Jérôme, "Porous poly $(\alpha$-hydroxyacid)/Bioglass composite scaffolds for bone tissue engineering. I: preparation and in vitro characterisation," Biomaterials, vol. 25, no. 18, pp. 4185-4194, 2004.

[123] S. J. Lee, J. Liu, S. H. Oh, S. Soker, A. Atala, and J. J. Yoo, "Development of a composite vascular scaffolding system that withstands physiological vascular conditions," Biomaterials, vol. 29, no. 19, pp. 2891-2898, 2008.

[124] M. Sato, Y. Nakazawa, R. Takahashi et al., "Small-diameter vascular grafts of Bombyx mori silk fibroin prepared by a combination of electrospinning and sponge coating," Materials Letters, vol. 64, no. 16, pp. 1786-1788, 2010.

[125] K. T. Shalumon, K. H. Anulekha, K. P. Chennazhi, H. Tamura, S. V. Nair, and R. Jayakumar, "Fabrication of chitosan/poly(caprolactone) nanofibrous scaffold for bone and skin tissue engineering," International Journal of Biological Macromolecules, vol. 48, no. 4, pp. 571-576, 2011.

[126] D. Yang, Y. Jin, Y. Zhou et al., "In situ mineralization of hydroxyapatite on electrospun chitosan-based nanofibrous scaffolds," Macromolecular Bioscience, vol. 8, no. 3, pp. 239-246, 2008.

[127] Y. S. Zhou, D. Yang, X. Chen, Q. Xu, F. Lu, and J. Nie, "Electrospun water-soluble carboxyethyl chitosan/poly(vinyl alcohol) nanofibrous membrane as potential wound dressing for skin regeneration," Biomacromolecules, vol. 9, no. 1, pp. 349354, 2008.

[128] Z. G. Wang, J. Q. Wang, and Z. K. Xu, "Immobilization of lipase from Candida rugosa on electrospun polysulfone nanofibrous membranes by adsorption," Journal of Molecular Catalysis B, vol. 42, no. 1-2, pp. 45-51, 2006.

[129] S. F. Li, J. P. Chen, and W. T. Wu, "Electrospun polyacrylonitrile nanofibrous membranes for lipase immobilization," Journal of Molecular Catalysis B, vol. 47, no. 3-4, pp. 117-124, 2007.

[130] Y. Wang and Y. L. Hsieh, "Immobilization of lipase enzyme in polyvinyl alcohol (PVA) nanofibrous membranes," Journal of Membrane Science, vol. 309, no. 1-2, pp. 73-81, 2008. 
[131] X. J. Huang, A. G. Yu, and Z. K. Xu, "Covalent immobilization of lipase from Candida rugosa onto poly(acrylonitrile-co-2hydroxyethyl methacrylate) electrospun fibrous membranes for potential bioreactor application," Bioresource Technology, vol. 99, no. 13, pp. 5459-5465, 2008.

[132] R. M. Schek, J. M. Taboas, S. J. Hollister, and P. H. Krebsbach, "Tissue engineering osteochondral implants for temporomandibular joint repair," Orthodontics-Craniofacial Research, vol. 8, no. 4, pp. 313-319, 2005.

[133] B. P. Chan and K. W. Leong, "Scaffolding in tissue engineering: general approaches and tissue-specific considerations," European Spine Journal, vol. 17, no. 4, supplement, pp. S467-S479, 2008.

[134] X. Miao, D. M. Tan, J. Li, Y. Xiao, and R. Crawford, "Mechanical and biological properties of hydroxyapatite/tricalcium phosphate scaffolds coated with poly(lactic-co-glycolic acid)," Acta Biomaterialia, vol. 4, no. 3, pp. 638-645, 2008.

[135] C. Erisken, D. M. Kalyon, and H. Wang, "Functionally graded electrospun polycaprolactone and $\beta$-tricalcium phosphate nanocomposites for tissue engineering applications," Biomaterials, vol. 29, no. 30, pp. 4065-4073, 2008.

[136] J. Zhou, C. Cao, X. Ma, and J. Jin, "Electrospinning of silk fibroin and collagen for vascular tissue engineering," International Journal of Biological Macromolecules, vol. 47, no. 4, p. 514, 2010.

[137] X. J. Huang, A. G. Yu, J. Jiang, C. Pan, J. W. Qian, and Z. $\mathrm{K}$. Xu, "Surface modification of nanofibrous poly(acrylonitrileco-acrylic acid) membrane with biomacromolecules for lipase immobilization," Journal of Molecular Catalysis B, vol. 57, no. 14, pp. 250-256, 2009.

[138] S. F. Li and W. T. Wu, "Lipase-immobilized electrospun PAN nanofibrous membranes for soybean oil hydrolysis," Biochemical Engineering Journal, vol. 45, no. 1, pp. 48-53, 2009.

[139] B. Liu, P. Li, C. L. Zhang, Y. Wang, and Y. S. Zhao, “Theanine synthesized by immobilizing Pseudomonas nitroreducens LY in nanofibrous membranes," Process Biochemistry, vol. 45, no. 8, pp. 1330-1333, 2010.

[140] X. Kang, C. Pan, Q. Xu et al., “The investigation of electrospun polymer nanofibers as a solid-phase extraction sorbent for the determination of trazodone in human plasma," Analytica Chimica Acta, vol. 587, no. 1, pp. 75-81, 2007.

[141] L. Wu, X. Zhang, and H. Ju, "Amperometric glucose sensor based on catalytic reduction of dissolved oxygen at soluble carbon nanofiber," Biosensors and Bioelectronics, vol. 23, no. 4, pp. 479-484, 2007.

[142] X. Wang, T. Yang, X. Li, and K. Jiao, “Three-step electrodeposition synthesis of self-doped polyaniline nanofiber-supported flower-like Au microspheres for high-performance biosensing of DNA hybridization recognition," Biosensors and Bioelectronics, vol. 26, no. 6, pp. 2953-2959, 2011.

[143] T. Yang, Y. Feng, W. Zhang, S. Ma, and K. Jiao, "Synergistic membrane of $\mathrm{ZrO}_{2}$ /self-doped polyaniline nanofibres fabricated by controllable electrodeposition for DNA hybridization detection," Journal of Electroanalytical Chemistry, vol. 656, no. 1-2, pp. 140-146, 2011.

[144] P. Gomathi, D. Ragupathy, J. H. Choi et al., "Fabrication of novel chitosan nanofiber/gold nanoparticles composite towards improved performance for a cholesterol sensor," Sensors and Actuators B, vol. 153, no. 1, pp. 44-49, 2011.

[145] Z. Du, C. Li, L. Li, M. Zhang, S. Xu, and T. Wang, "Simple fabrication of a sensitive hydrogen peroxide biosensor using enzymes immobilized in processable polyaniline nanofibers/chitosan film," Materials Science and Engineering C, vol. 29, no. 6, pp. 1794-1797, 2009.

[146] Y. Tan, J. Kan, and S. Li, "Amperometric biosensor for catechol using electrochemical template process," Sensors and Actuators B, vol. 152, no. 2, pp. 285-291, 2011.

[147] Y. Gao, X. Li, J. Gong, B. Fan, Z. Su, and L. Qu, "Polyaniline nanotubes prepared using fiber mats membrane as the template and their gas-response behavior," Journal of Physical Chemistry C, vol. 112, no. 22, pp. 8215-8222, 2008.

[148] N. J. Pinto, I. Ramos, R. Rojas, P. C. Wang, and A. T. Johnson, "Electric response of isolated electrospun polyaniline nanofibers to vapors of aliphatic alcohols," Sensors and Actuators $B$, vol. 129, no. 2, pp. 621-627, 2008.

[149] O. Landau, A. Rothschild, and E. Zussman, "Processingmicrostructure-properties correlation of ultrasensitive gas sensors produced by electrospinning," Chemistry of Materials, vol. 21, no. 1, pp. 9-11, 2009.

[150] J. Zhang, J. Lei, Y. Liu, J. Zhao, and H. Ju, "Highly sensitive amperometric biosensors for phenols based on polyanilineionic liquid-carbon nanofiber composite," Biosensors and Bioelectronics, vol. 24, no. 7, pp. 1858-1863, 2009.

[151] X. Wang, C. Drew, S. H. Lee et al., "Electrospun nanofibrousmembranes for highly sensitive optical sensors," Nano Letters, vol. 2, no. 11, pp. 1273-1275, 2002.

[152] X. Wang, Y. G. Kim, C. Drew, B. C. Ku, J. Kumar, and L. A. Samuelson, "Electrostatic assembly of conjugated polymer thin layers on electrospun nanofibrous membranes for biosensors," Nano Letters, vol. 4, no. 2, pp. 331-334, 2004.

[153] C. Jianrong, M. Yuqing, H. Nongyue, W. Xiaohua, and L. Sijiao, "Nanotechnology and biosensors," Biotechnology Advances, vol. 22, no. 7, pp. 505-518, 2004.

[154] K. Ramanathan, M. A. Bangar, M. Yun et al., "Bioaffinity sensing using biologically functionalized conducting-polymer nanowire," Journal of American Chemical Society, vol. 127, no. 2, pp. 496-497, 2005.

[155] N. Kattamuri, J. H. Shin, B. Kang et al., "Development and surface characterization of positively charged filters," Journal of Materials Science, vol. 40, no. 17, pp. 4531-4539, 2005.

[156] S. Ramakrishna, K. Fujihara, W. E. Teo, T. Yong, Z. Ma, and R. Ramaseshan, "Electrospun nanofibers: solving global issues," Materials Today, vol. 9, no. 3, pp. 40-50, 2006.

[157] C. Burger, B. S. Hsiao, and B. Chu, "Nanofibrous marterials and their applications," Annual Review of Materials Research, vol. 36, pp. 333-368, 2006.

[158] E. H. Jeong, J. Yang, and J. H. Youk, "Preparation of polyurethane cationomer nanofiber mats for use in antimicrobial nanofilter applications," Materials Letters, vol. 61, no. 18, pp. 3991-3994, 2007. 

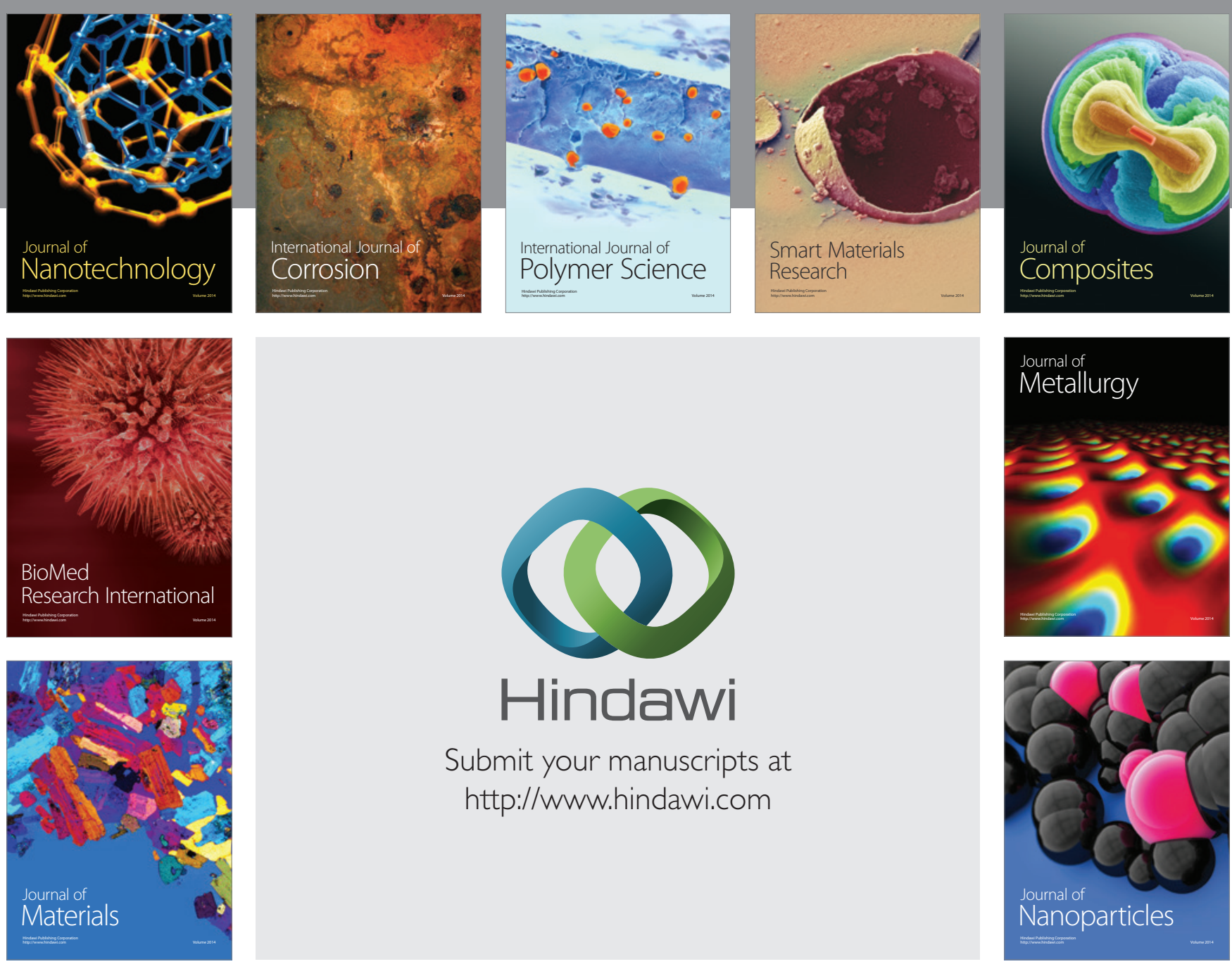

Submit your manuscripts at http://www.hindawi.com
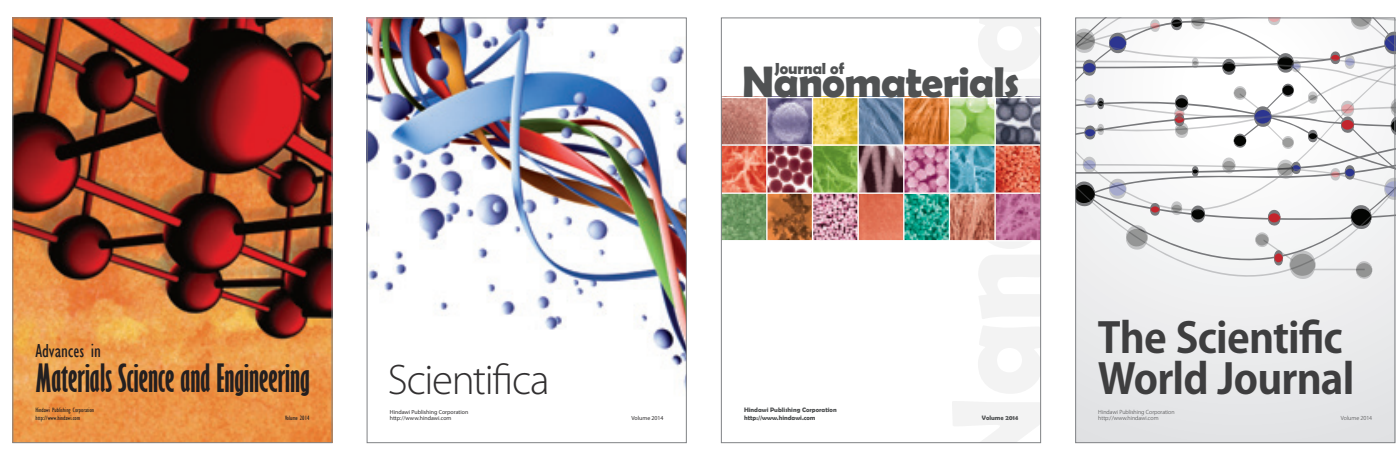

\section{The Scientific World Journal}
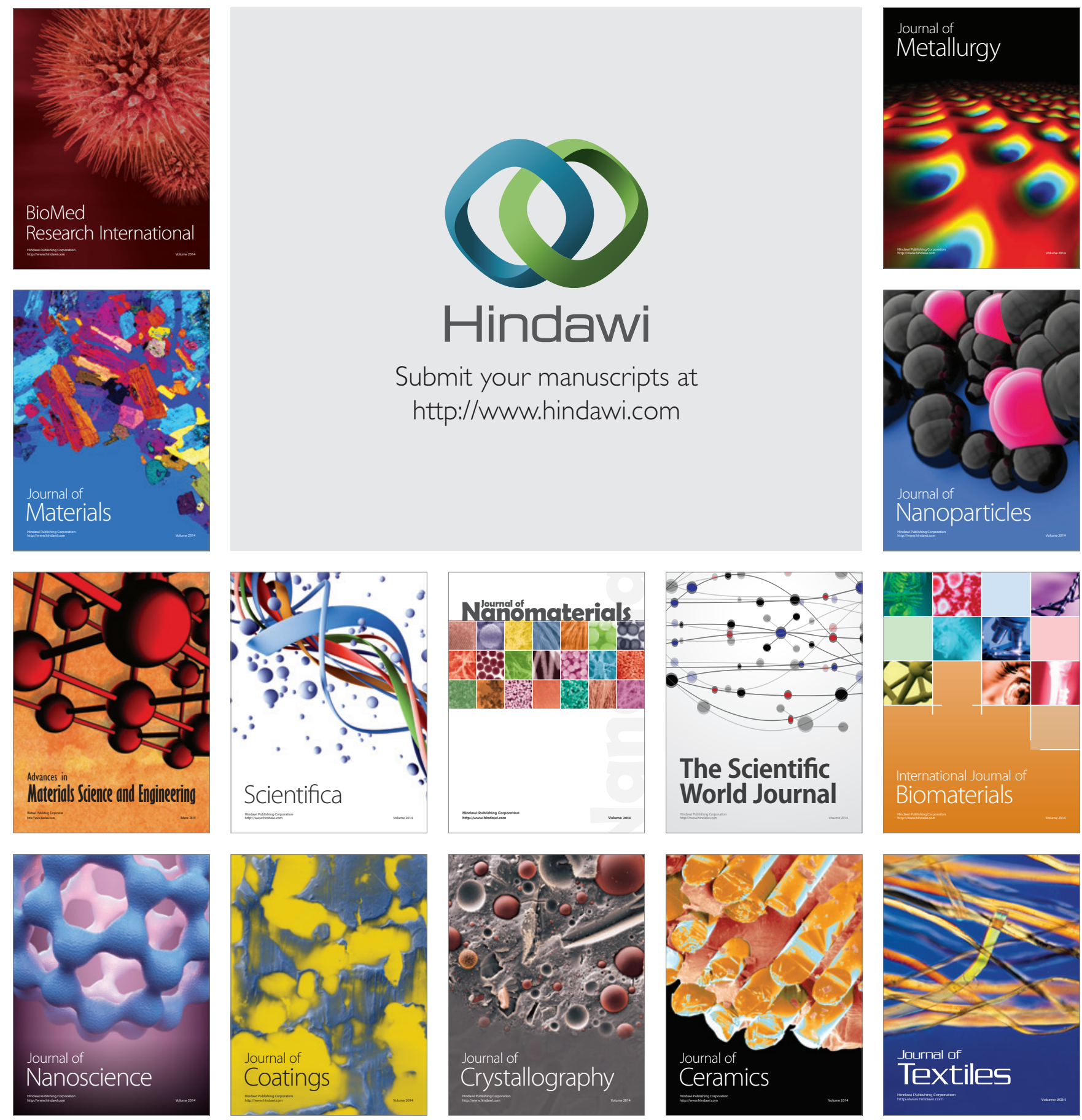